\title{
Photonic-Crystal Josephson Traveling-Wave Parametric Amplifier
}

\author{
Luca Planat๑, ${ }^{1}$ Arpit Ranadive, ${ }_{1}^{1}$ Rémy Dassonneville, ${ }^{1}$ Javier Puertas Martínez, ${ }^{1}$ Sébastien Léger, ${ }^{1}$ Cécile Naud, ${ }^{1}$ \\ Olivier Buisson, ${ }^{1}$ Wiebke Hasch-Guichard, ${ }^{1}$ Denis M. Basko, ${ }^{2}$ and Nicolas Roch ${ }^{1, *}$ \\ ${ }^{1}$ Université Grenoble Alpes, CNRS, Grenoble INP, Institut Néel, 38000 Grenoble, France \\ ${ }^{2}$ Université Grenoble Alpes, CNRS, LPMMC, 38000 Grenoble, France
}

(Received 20 September 2019; revised manuscript received 4 January 2020; accepted 3 March 2020; published 28 April 2020)

\begin{abstract}
A microwave amplifier combining noise performances as close as possible to the quantum limit with large bandwidth and high saturation power is highly desirable for many solid-state quantum technologies. Here, we introduce a new traveling-wave parametric amplifier based on superconducting quantum interference devices. It displays a 3-GHz bandwidth, a $-100-\mathrm{dBm}$ saturation (1-dB compression) point and added noise near the quantum limit. Compared to the previous state of the art, it is an order of magnitude more compact, its characteristic impedance is in situ tunable, and its fabrication process requires only two lithography steps. The key is the engineering of a gap in the dispersion relation of the transmission line. This is obtained using a periodic modulation of the SQUID size, similarly to what is done with photonic crystals. Moreover, we provide a new theoretical treatment to describe the nontrivial interplay between nonlinearity and such periodicity. Our approach provides a path to cointegration with other quantum devices such as qubits given the low footprint and easy fabrication of our amplifier.
\end{abstract}

DOI: 10.1103/PhysRevX.10.021021

\section{INTRODUCTION}

A wide range of quantum technologies relies on the faithful detection of microwave signals at millikelvin temperatures. This includes quantum computing with superconducting [1] and spin [2] qubits, electron spin resonance with quantum-limited sensitivity [3], sensing the motion of nanomechanical resonators [4], detecting cosmic microwave background using kinetic inductance detectors (KID) [5], or the quest for axionic dark matter [6]. For all these applications, sensitivity is limited by the first amplifier of the detection chain. When this level of performance is needed, Josephson parametric amplifiers (JPA) $[7,8]$ are considered the technology of choice since they can reach the quantum limit of amplification [9-12]. These amplifiers are built around the parametric interaction between three waves called signal, pump, and idler. However, JPA's are still insufficient for demanding applications such as a multiplexed readout of qubits $[13,14]$ or KID arrays [15] where both large bandwidth and high saturation power are required. As a consequence, many works focused on these

\footnotetext{
*Corresponding author. nicolas.roch@neel.cnrs.fr

Published by the American Physical Society under the terms of the Creative Commons Attribution 4.0 International license. Further distribution of this work must maintain attribution to the author(s) and the published article's title, journal citation, and DOI.
}

Subject Areas: Condensed Matter Physics, Mesoscopics, Quantum Information two figures of merit in recent years. Bandwidth can be largely improved using impedance matching techniques [16-18], while reducing unwanted nonlinear effects was key to obtaining larger saturation powers [19-23].

An alternative to JPA's is to engineer a nonresonant, nonlinear device, such as a nonlinear transmission line, where the amplification arises step by step, as was already extensively demonstrated in the optical domain [24] or with traveling-wave tube amplifiers [25]. Such devices are dubbed traveling-wave parametric amplifiers (TWPA) [26]. They are intrinsically broadband since they do not rely on a resonant structure. In addition, the distributed nature of their nonlinearity (the gain is not provided by one unique unit cell) is also very valuable to improve dynamical range, as was demonstrated for JPA's [19,22,23,27,28]. Wide bandwidth and high saturation power combined with low noise approaching the quantum limit make them very attractive to any microwave measurement setup. However, such appealing figures of merit come at a price. This nonlinear transmission line should be impedance matched to $50 \Omega$ to avoid unwanted reflections, synonymous of gain ripples. Moreover, not only should the energy between signal, pump, and idler waves be conserved during the parametric process but the momentum must be conserved as well since these are traveling waves. This latter condition, also known as phase matching [24], is automatically fulfilled in a resonant device but is far less trivial to obtain in a traveling-wave structure. Indeed, the nonlinearity at the heart of the amplification process also causes a nonlinear 
phase mismatch between the signal, idler, and pump waves, which limits the gain and bandwidth of the amplifier.

The first demonstration of a TWPA in the microwave domain relied on the nonlinearity of NbTiN [29], a high kinetic inductance superconductor. The impedance matching was obtained using tapers while the phase matching was enforced via periodic loading structures, similarly to what is done with photonic crystals [30-34]. However, such high kinetic inductance TWPA's (KTWPA's) show very small dispersion outside of the band of the periodic loading structures since their only cutoff frequency is the superconducting gap, located at frequencies as high as several hundreds of gigahertz. This might give rise to shock waves [35] and backward amplification [36]. Moreover, the nonlinearity is intrinsically weak in such materials [37-39]. As a consequence, KTWPA's require very high pump powers, which can cause unwanted heating effects [29]. These two limitations probably explain why it is only very recently that KTWPA's reached the quantum limit [40].

The first quantum-limited TWPA [41] was a nonlinear transmission line made from a Josephson-junction metamaterial [42-44]. To achieve impedance matching, the high inductance of Josephson junctions was compensated by adding a discrete planar shunt capacitance to each unit cell. The phase-matching issue was resolved using an elegant but technologically challenging resonant technique $[45,46]$. This design still sets the state of the art in terms of gain, bandwidth, and noise performance but requires a discouragingly complicated fabrication overhead associated with multilayer lithography involving different metals. Moreover, this amplifier cannot be directly integrated with superconducting qubits since it relies on a niobium trilayer fabrication process [47].

In this article, we introduce a new microwave superconducting quantum interference device (SQUID) based traveling-wave parametric amplifier (STWPA). It combines near quantum-limited noise performances with a simple design and fabrication process involving only two lithography steps [48], a small footprint, direct integration capability with superconducting qubits and in situ flux tunability. Our device consists of SQUID metamaterial [11,49], which is subsequently covered by a thin dielectric and a top ground plane [see Fig. 1(a)]. This simple approach allows us to solve the impedance matching issue. Moreover, contrary to previous TWPA's, this impedance can now be tuned in situ since a SQUID can be operated as a tunable inductor controlled by a dc magnetic flux $\Phi$. To resolve the phase-matching problem, we engineer the dispersion relation of this nonlinear transmission line using a spatial modulation of its impedance $[29,33]$ by periodically changing the SQUID size within the metamaterial [50,51]. This periodic modulation opens a gap in the dispersion relation [52], similar to what is observed for photonic crystals or for Bloch waves in crystal lattices [see Figs. 1(b) and 1(d)] [53]. In order to compensate for the
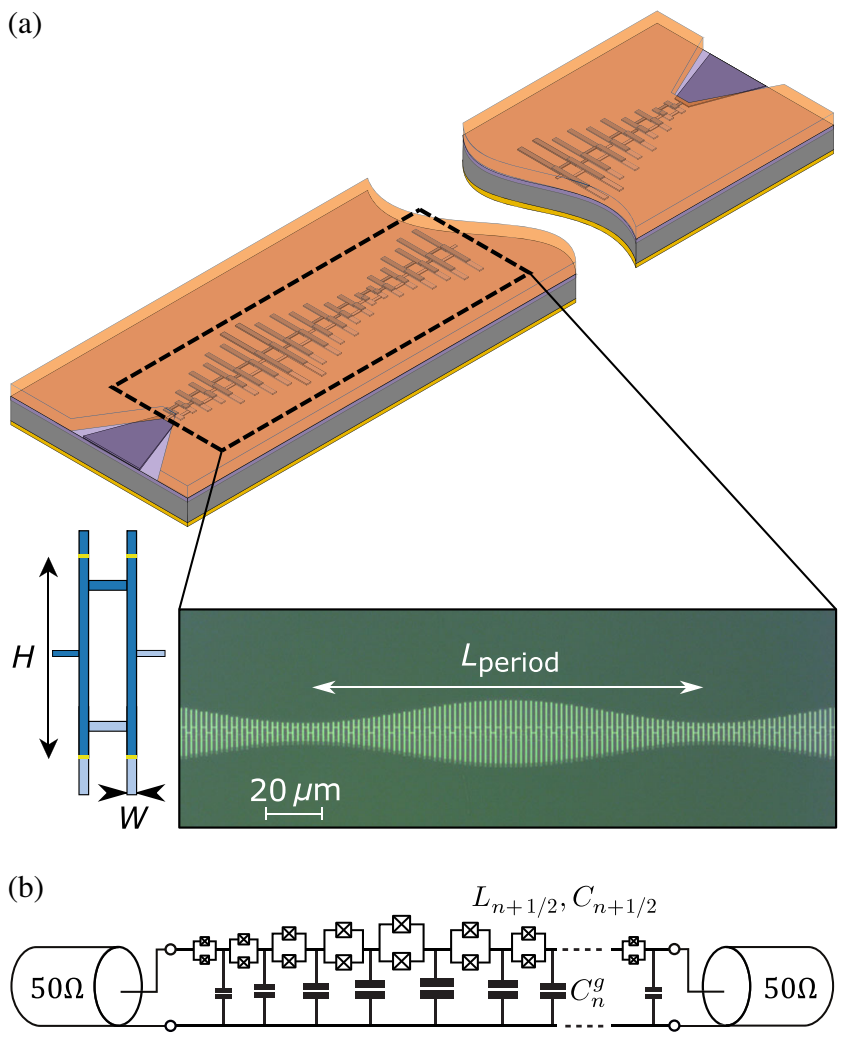

(c)

(d)
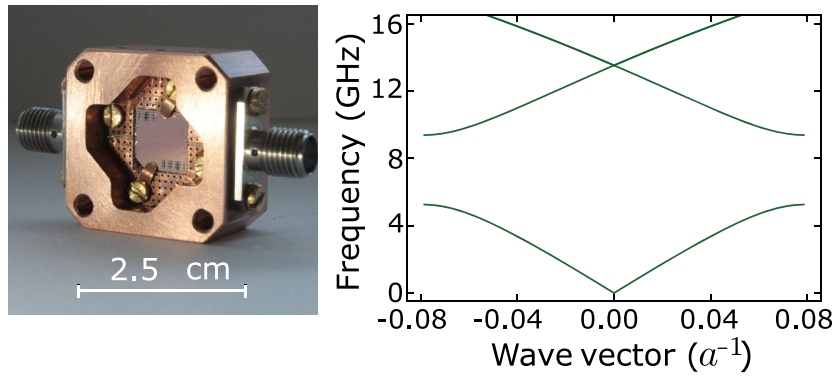

FIG. 1. (a) Schematic of the photonic crystal STWPA. The spatially modulated SQUID array is fabricated on a silicon substrate. SQUID are then covered by a thin alumina layer (purple, obtained via atomic-layer deposition) and a thick copperlayer orange, which is used as a top metallic ground. Schematic: representation of one SQUID. $W$ denotes the junction width while $H$ is the height of the junction, which is the overlap between the top (dark blue) and bottom (light blue) aluminum layers. More details in Sec. A. Optical picture: two periods of the SQUID array. Only the height is modulated. The modulation amplitude on the figure $(\gamma=60 \%)$ is greatly exaggerated for the sake of presentation; the actual samples have $\gamma=4 \%$ and $2 \%$. (b) Electrical sketch of the STWPA. The electrical parameters of the unit cells are following a sinusoidal modulation. (c) Picture of the sample. The chip is the mauve square in the middle, plugged to a copper printed circuit board in its copper box. The magnetic field is applied normal to the chip using a coil directly anchored onto the copper box. (d) Dispersion relation, plotted in the first Brillouin zone, of an array of 2160 SQUID with realistic microscopic parameters and a modulation amplitude $\gamma=60 \%$. 
nonlinear phase matching intrinsic to the amplification process, the pump frequency is adjusted close to this gap to take advantage of the local distortion of the dispersion relation [29]. Contrary to KTWPA, our device benefits from a well-controlled and stronger nonlinearity $[54,55]$ and a cut-off or plasma frequency around $30 \mathrm{GHz}$, which prevents unwanted nonlinear phenomena.

For quantitative understanding of the amplification process in our device we develop a novel self-consistent theory of the nonlinear pump propagation in the photonic crystal. When the pump wave propagates close to the edge of the photonic gap, it changes the properties of the photonic crystal (via the self-Kerr effect), modifies the spectral gap position, and thus the pump intensity which enters the STWPA. Even for a weak spatial modulation and weak pump power, this effect is nonperturbative. The selfconsistent description is crucial for quantitative modeling of the measured gain profile.

\section{DESCRIPTION OF THE DEVICE}

In first a lithography step, we pattern an array of $N_{J}$ SQUID's. To match this array to $50-\Omega$ characteristic impedance without adding extra components, such as large discrete capacitors, we cover the array with a very thin dielectric layer (alumina, $28 \mathrm{~nm}$ ) and a thick copper layer to obtain a very close ground plane resulting in very high ground capacitance per unit length. We choose a nonsuperconducting metal for the ground in order to avoid flux trapping and effects of Meissner currents when an external magnetic field threads the SQUID loops. A copper thickness of $1 \mu \mathrm{m}$ is enough to avoid significant conductor loss coming from the ground plane [48]. We cannot completely avoid the dielectric loss, as observed in most travelingwave structures [41]. These dielectric losses are both frequency and power dependent (for example, 5-dB attenuation is found at $6 \mathrm{GHz}$ and very low signal power). In a second lithography step, we pattern openings in this ground plane to allow proper wire bonding, as shown in Fig. 1(a). The obtained ground capacitance is a few tens of femtofarads per SQUID using this simple microstrip geometry [48]. Finally, the absence of large capacitors for impedance matching and resonators for phase matching makes this STWPA very compact with a footprint of $7.2 \mathrm{~mm}$ by $13 \mu \mathrm{m}$ [see Figs. 1(a) and 1(c)].

The two Josephson junctions forming each SQUID have a constant nominal width $W=0.45 \mu \mathrm{m}$ and a periodically modulated height $H$, as schematically shown in Fig. 1(a) (see the Appendix A for details on the geometry). The nominal mean height is $\bar{H}=12 \mu \mathrm{m}$. We choose sinusoidal modulations since closed-form expressions regarding the amplitude and the position of the gap exist in this case [50]. Here, we label the superconducting islands by an integer $n=0,1, \ldots, N_{J}$, so the SQUID connecting neighboring islands $n$ and $n+1$ is labeled by a half-integer $n+1 / 2$. We
TABLE I. Parameters of samples $A$ and $B$. The main difference is the modulation amplitude $\gamma$, which reflects directly in the width of their photonic gap.

\begin{tabular}{lcc}
\hline \hline Device & $A$ & $B$ \\
\hline Total SQUID number $N_{J}$ & 2160 & 2184 \\
Length per SQUID $a(\mu \mathrm{m})$ & 3.3 & 3.3 \\
Josephson capacitance $\bar{C}(\mathrm{fF})$ & 485 & 485 \\
Ground capacitance $\bar{C}^{g}(\mathrm{fF})$ & 42.6 & 42.6 \\
Zero-flux inductance $\bar{L}(p \mathrm{H})$ & $60.5 \pm 1.1$ & $61.7 \pm 1.1$ \\
Josephson modulation amplitude $\gamma$ & $4 \%$ & $2 \%$ \\
Ground capacitance modulation & $3.2 \%$ & $1.6 \%$ \\
$\quad$ amplitude $\zeta$ & & \\
Modulation period $N_{p}$ & 40 & 42 \\
Gap position $(\mathrm{GHz})$ & 7.45 & 7.15 \\
Gap width $(\mathrm{MHz})$ & 350 & 200 \\
\hline \hline
\end{tabular}

denote by $a$ the average length per SQUID, so that $N_{J} a$ is the physical length of the array.

If $N_{p}$ is the number of SQUIDs per period, $G=2 \pi / N_{p}$ is the reciprocal lattice vector (in units of $a^{-1}$ ) corresponding to the modulation. Both the critical current of each SQUID $n+1 / 2$ (inversely proportional to its Josephson inductance $L_{n+1 / 2}$ ) and the SQUID capacitance $C_{n+1 / 2}$ are proportional to the junction area. The capacitance $C_{n}^{g}$ between each island $n$ and the ground plane is proportional to the island area, and each island participates in two SQUID's. Thus, the modulated circuit parameters can be written as

$$
\begin{aligned}
L_{n+1 / 2}^{-1} & =\bar{L}^{-1}[1+\gamma \cos G(n+1 / 2)], \\
C_{n+1 / 2} & =\bar{C}[1+\gamma \cos G(n+1 / 2)], \\
C_{n}^{g} & =\bar{C}^{g}\left[1+\frac{\zeta}{2} \sum_{ \pm} \cos G(n \pm 1 / 2)\right],
\end{aligned}
$$

where $\bar{L}, \bar{C}$, and $\bar{C}^{g}$ are the values corresponding to the average SQUID height while $\gamma$ and $\zeta$ are modulation amplitudes of the Josephson parameters $(L$ and $C$ ) and the ground capacitance, respectively. The SQUID plasma frequency $1 / \sqrt{L_{n+1 / 2} C_{n+1 / 2}}=1 / \sqrt{\bar{L} \bar{C}}$ remains constant along the chain. This article presents two devices, labeled $A$ and $B$, whose parameters are listed in Table I. In our case, $\zeta$ is close to $\gamma$. All parameters are determined from the geometry (see Appendix A for details), except the Josephson inductance $\bar{L}$ which is found by fitting the experimental dispersion relation in the linear region, as discussed in the next section.

To model the device theoretically, we use the classical equations of motion for the superconducting phases $\phi_{n}$ on each island $n$,

$$
\begin{aligned}
& \frac{d^{2}}{d t^{2}}\left[C_{n}^{g} \phi_{n}+C_{n+1 / 2}\left(\phi_{n}-\phi_{n+1}\right)+C_{n-1 / 2}\left(\phi_{n}-\phi_{n-1}\right)\right] \\
& \quad+\frac{\sin \left(\phi_{n}-\phi_{n+1}\right)}{L_{n+1 / 2}}+\frac{\sin \left(\phi_{n}-\phi_{n-1}\right)}{L_{n-1 / 2}}=0 .
\end{aligned}
$$


The linear dispersion is obtained by plugging into these equations the modulated parameters as given by Eq. (1), expanding $\sin \theta \approx \theta$, and looking for the solution in the form

$$
\phi_{n}(t)=\left(A e^{i k n}+B e^{i(k-G) n}\right) e^{-i \omega t}+\text { c.c. }
$$

The nonlinear pump propagation and the gap shift are determined by expanding $\sin \theta \approx \theta-\theta^{3} / 6$ in Eqs. (2), substituting solution (3), retaining only terms proportional to $e^{ \pm i(k n-\omega t)}, e^{ \pm i(k n-G n-\omega t)}$, and solving self-consistently for $A, B, k$ for a given incident pump power (see Appendix B for details). The signal amplification is obtained by adding weak signal and idler waves $\phi_{n}^{s} e^{-i \omega_{s} t}, \phi_{n}^{i} e^{-i \omega_{i} t}$ on top of the previously determined pump solution, linearizing Eqs. (2) with respect to $\phi_{n}^{s, i}$, and solving the resulting linear equations directly, without any a priori assumptions on the spatial dependence of $\phi_{n}^{s, i}$.

\section{TRANSMISSION IN A NONLINEAR PHOTONIC CRYSTAL}

We first measure the dispersion relation in the linear regime without the pump tone and at very low signal power. The wave vector $k$ is obtained by measuring the phase along the STWPA, dividing by its full length $L_{\mathrm{TWPA}}=N_{J} a$, and subtracting the phase measured with a dummy $50 \Omega$ line from it (see Appendix C). The $2 \pi$ uncertainty introduced by the absence of a signal for very low frequencies is lifted by following the procedure described in [41]. Namely, we fitted the linear region at low frequencies with an affine law in order to get the intercept, which is an integer multiple of $2 \pi$. We then substract this intercept. Once this calibration is done, we can fit the dispersion relation with our model, leaving $\bar{L}$ as the only fit parameter, while the rest of the parameters $\left(\gamma, N_{J}, N_{p}\right.$, $\bar{C}, \bar{C}^{g}$ ) are set by the design and the fabrication process. We observe a quasilinear dependence within the $3-9 \mathrm{GHz}$ band and a kink around $7 \mathrm{GHz}$ corresponding to the photonic gap [see Fig. 2(a)]. The inset of Fig. 2(a) shows an enlargement on the gap region of sample $A$. The gap is located near $7.25 \mathrm{GHz}$ and is about $350 \mathrm{MHz}$ wide. In the rest of the article, all microscopic and fabrication parameters are fixed.

Although there is an overall good agreement between theory and experimental data, there is a little discrepancy regarding the exact wave vector and frequency position of the gap. According to the theory, its wave vector position should be $G / 2=7.85 \times 10^{-2}$ and its frequency $7.29 \mathrm{GHz}$. Experimentally, we find $7.80 \times 10^{-2}$ and $7.24 \mathrm{GHz}$, which is a deviation of $0.6 \%$ and $0.7 \%$ from the expected value, respectively. This should be understood as the precision of our experimental method to obtain the dispersion relation. To obtain a perfect frequency match of the gap position, we allow up to $1.75 \% \bar{L}$ variation. (a)
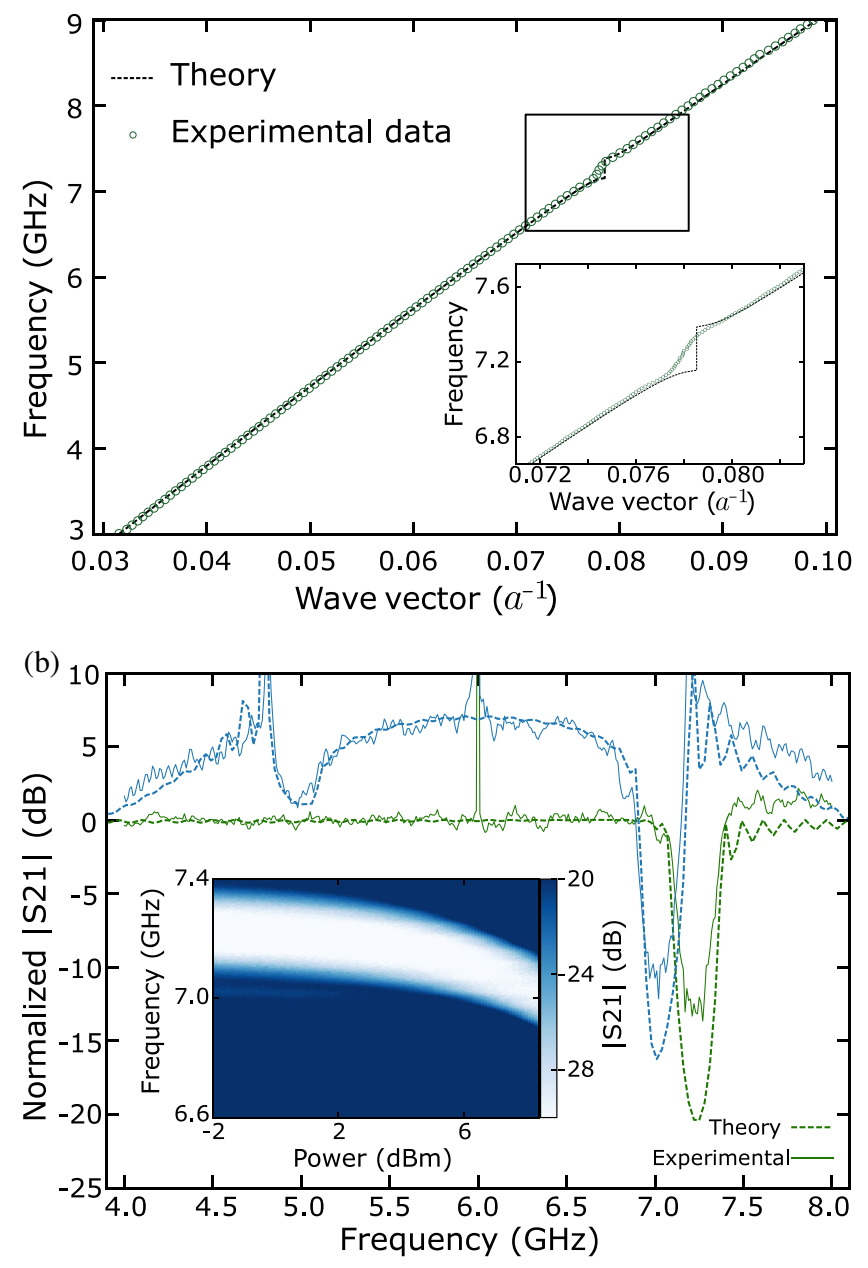

FIG. 2. (a) Dispersion relation of sample $A$ measured at a very low magnetic flux $\left(\Phi / \Phi_{0}=0.1\right)$. The theoretical curve is a fit to the linear dispersion relation with $\bar{L}$ as a fit parameter. The kink at $7 \mathrm{GHz}$ is the photonic gap created by spatial modulation. Inset: Close-up of the photonic gap. Theory agrees with experimental data and reproduces the gap position with a $0.6 \%$ error. (b) Normalized probe transmission of sample $A$ for a signal power set to $P_{s}=-135 \mathrm{dBm}$ and for different pump powers $\left(\omega_{P} / 2 \pi=6 \mathrm{GHz}\right.$, green: $P_{P}=-83.8 \mathrm{dBm}$, blue: $\left.P_{P}=-70.3 \mathrm{dBm}\right)$. The gap is shifted by more than its width at large pump power. Inset: Color map of the probe transmission versus probe frequency and pump power.

An important feature of this photonic crystal STWPA is the dependence of the gap position on the incident pump power. Understanding this nonlinear shift is of prime importance since the pump frequency needs to be precisely adjusted close to the gap whose frequency is shifted by the pump power at the same time. There are two ways to investigate this effect. The first one, that we call self-shift, consists of sending an intense microwave tone around the gap frequency and measuring transmission. Alternatively, we can infer the cross shift by pumping the STWPA far from the gap and by measuring its transmission using a 


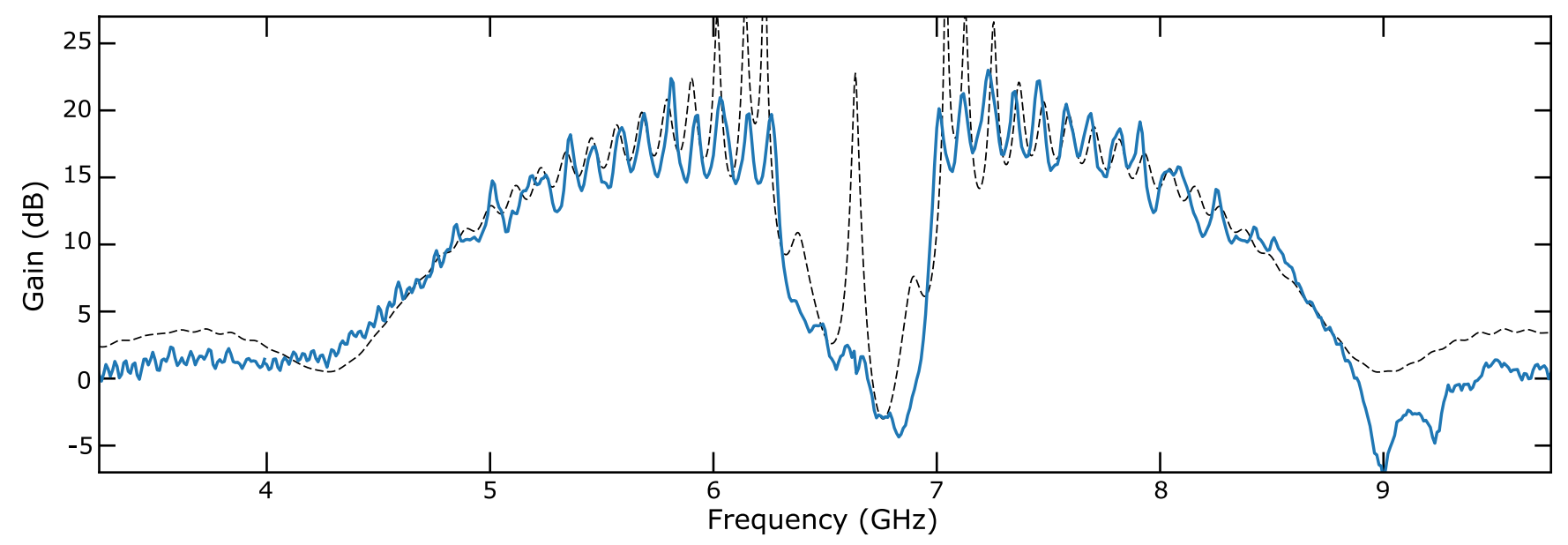

FIG. 3. Gain profile of the STWPA at magnetic flux $\Phi / \Phi_{0}=0.2$ and pump frequency $6.635 \mathrm{GHz}$ and signal power $P_{s}=-117 \mathrm{dBm}$. The theoretical gain profile (dashed black line) is obtained for a pump power $P_{\text {input }}=-70.2 \mathrm{dBm}$.

probe tone. Experimentally, it is more difficult to access the self-shift since the measurement time has to be matched to the intrinsic dynamics of this nonperturbative phenomena, in a way similar to what is done for JPA close to bifurcation [56]. Hence, we focused on cross-shift measurements.

The probe transmission while STWPA is pumped at $6 \mathrm{GHz}$ is shown on Fig. 2(b). At low pump power we recover the linear transmission. At high power, we observe up to $7 \mathrm{~dB}$ gain and a $-3-\mathrm{dB}$ bandwidth of $1.5 \mathrm{GHz}$. These small gains and bandwidths are the consequences of poor phase matching since the pump does not benefit from the dispersive feature provided by the gap. More interestingly, we observe a large shift of the gap frequency position compared to the low pump-power case. This nonlinear shift has the same order of magnitude as the actual gap width (few hundreds of megahertz).

A comparison between data and theory is done by setting all the parameters of the metamaterial to the values obtained by the dispersion relation analysis. The only free parameter is the pump power. Since this model reproduces successfully the nonlinear behavior of our STWPA over a broad pump-power range, it provides an accurate method to calibrate the attenuation of the input line. The calibration of the input attenuation line has been carried out by taking advantage of the large cross-shift of the gap frequency position with respect to the pump power. We compared the experimental pump power sent from the rf source at room temperature to the theoretical pump power at the input of the STWPA needed to make the photonic gap shift from its initial frequency. The difference between the experimental "hot" pump power and the theoretical "cold" pump power gives an attenuation of $78.8 \mathrm{~dB}$ between the rf source output and the STWPA input. This value is the sum of the input line attenuation $A_{\text {system }}$ and the attenuation of the pump amplitude within the STWPA $A_{\text {TWPA }}$. Given the small loss of the STWPA, it is taken as position independent [41], such that $A_{\mathrm{TWPA}}=\exp \left(k_{p}^{\prime \prime} N_{J} a\right)$, where $k_{p}^{\prime \prime}=\left(k_{p}^{\prime} \tan \delta\right) / 2$ is the imaginary part of the complex wave vector (in the limit of small losses), $N_{J} a$ is the total length of the STWPA, and $\tan \delta$ is the loss tangent. We find $A_{\mathrm{TWPA}}=3.7 \mathrm{~dB}$ at $6 \mathrm{GHz}$. This result, giving $A_{\text {system }}=75.1 \mathrm{~dB}$, is in good agreement with the system attenuation calculated at $6 \mathrm{GHz}$ by summing all the discrete attenuators $(63.0 \mathrm{~dB})$, power splitter $(6.8 \mathrm{~dB})$, filter $(2.0 \mathrm{~dB})$, cryogenic temperature cables $(80 \mathrm{~cm}, 1.5 \mathrm{~dB})$ and room-temperature cables $(2.3 \mathrm{~dB})$, giving $75.6 \mathrm{~dB}$ and leading to a discrepancy of $0.5 \mathrm{~dB}$, which can be seen as the uncertainty of our calibration method. We then decide to set the system attenuation to $A_{\text {system }}=75.1 \mathrm{~dB} \pm$ $0.5 \mathrm{~dB}$. Attenuation calibration is described in detail in Appendix D.

\section{PHASE-MATCHED AMPLIFICATION}

We now report on the characteristics of the STWPA when pumped close to the edge of the photonic band gap (Fig. 3). Larger gain and bandwidth are expected since the nonlinear phase shift is now compensated. Finding the correct pump power and frequency is slightly trickier than for resonant phase matching Josephson traveling-wave parametric amplifier $[41,46]$ since the gap moves when pump power is changed. However, this issue is easy to overcome: similarly to current pumped JPA, the optimal pump configuration (frequency and power) must be found incrementally. Whenever power is increased, the frequency must be lowered to compensate its own backaction until optimal gain is reached.

With sample $A$, we obtain a mean maximum gain around $18 \mathrm{~dB}$ over a 3-GHz bandwidth, from which $700 \mathrm{MHz}$ must be taken out given the photonic gap. We also observe up to 5-dB gain ripples. Theoretical and experimentally achieved gain are shown in Fig. 3. This gain profile was taken with configuration (1) (see Appendix C for more details). Here, attenuation was again the only free parameter 

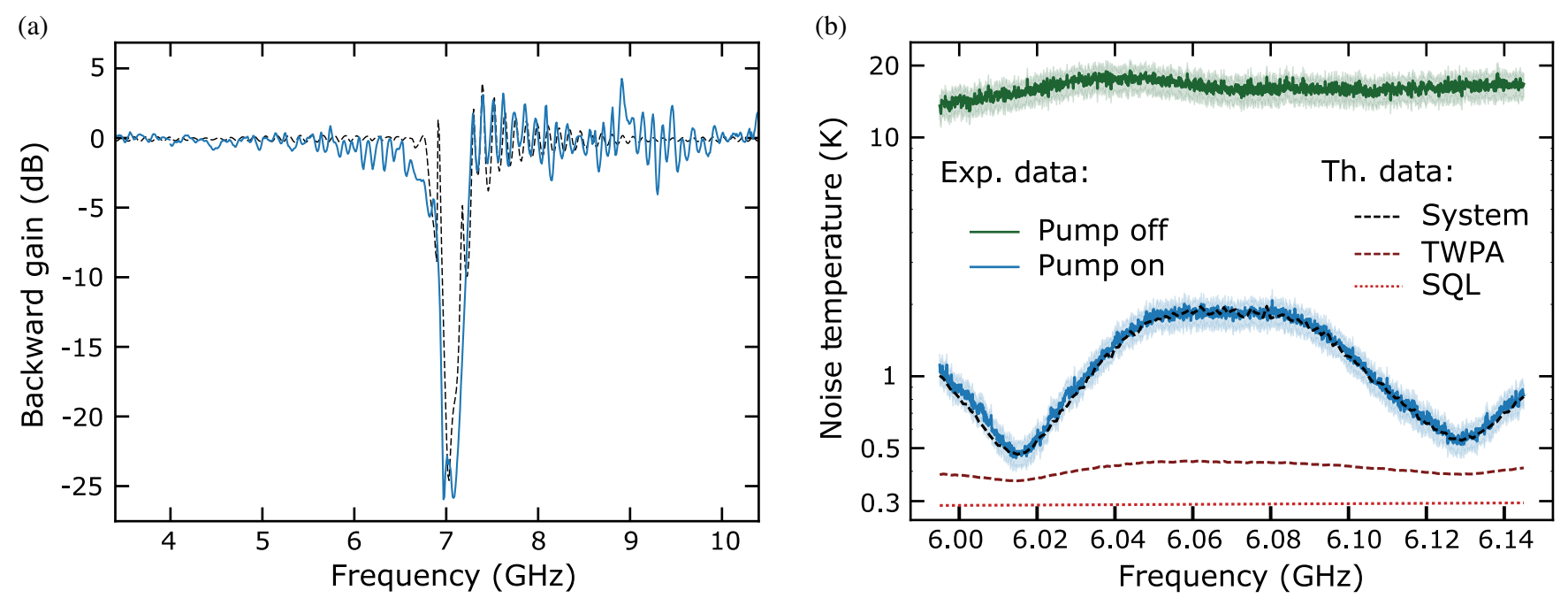

FIG. 4. (a) Backward gain measured with $\Phi / \Phi_{0}=0.1$ and pump frequency $6.9108 \mathrm{GHz}$. Expected gain is plotted for a pump power $P_{\text {input }}=-71.4 \mathrm{dBm}$. (b) Noise temperature of the STWPA. Measured PSD normalized by the system gain when the STWPA pump is off (green solid line, the light green area corresponds to error bars) or on (blue solid line, the light blue area corresponds to error bars). The red dotted line corresponds the standard quantum limit (SQL) of noise. The brown dashed line refers to the intrinsic noise of the STWPA, which is slightly above the SQL because of internal losses. The black dashed line is the total calculated system noise temperature. It is higher than the STWPA intrinsic noise temperature because of the finite STWPA gain. Noise ripples are caused by STWPA gain ripples. Theory curves also have error bars since they rely as well on a careful calibration of the total system gain. For the brown dashed line, it is between $40 \mathrm{mK}$ (gain peak) and $50 \mathrm{mK}$ (gain dip). For the black dashed line, it is between 60 and $260 \mathrm{mK}$.

$(79.5 \mathrm{~dB}$ at $6.6 \mathrm{GHz})$ and the measured gain is in very good agreement with theory. Sample $B$ shows smaller gain and bandwidth [see Sec. VI] because of its smaller gap. This feature is again well captured by our theoretical formalism. A qualitative explanation is that a small curvature around the gap can only provide a limited correction to the nonlinear phase shift induced by the strong pump [46]. In the present case, the curvature and size of the gap are directly linked since we use a simple sinusoidal modulation. However, in principle, a more advanced gap engineering could allow a more pronounced curvature without a larger gap size, which reduces the usable bandwidth of the amplifier. With sample $B$, we observe about $1 \mathrm{~dB}$ gain ripples. For the same maximum gain $(\sim 15 \mathrm{~dB})$, sample $A$ displays about the same ripples amplitude. We therefore believe these ripples come from impedance mismatches (see Appendix B 3), most likely localized between the subminiatures A connectors of the sample holder and the sample itself.

We further investigate the behavior of our STWPA by probing backward amplification, that is, amplification when signal and pump propagate in opposite directions. Backward amplification could potentially happen in periodic structures such as our STWPA and could be detrimental to the behavior of the device, creating gain ripples and reducing threshold for parametric oscillations [36,45]. But more importantly this could affect the STWPA directionality and thus create a strong backaction on the measured devices.

Choosing a conservative approach, we optimize the pumping parameters to maximize backward amplification.
Probing backward gain is carried out with configuration (2), discussed in Appendix C. This configuration allows us to send the STWPA pump in one direction while measuring the signal propagating in the opposite direction. We first probe the signal gain in the standard way (pump and signal have the same propagation direction) to get an optimal set of pump frequency $\Omega_{\text {opti }}$ and power $P_{\text {opti }}$, giving $\bar{G}_{\text {opti }}=18 \mathrm{~dB}$. Pump tone is then sent through the STWPA output while keeping the pump frequency fixed to $\Omega_{\text {opti }}$. Signal gain is then probed while sweeping pump power, since attenuation from one line to another can change.

The highest backward gain we could obtain is shown in Fig. 4(a) and stays below $5 \mathrm{~dB}$. We attribute this small value to two features of our STWPA. First, we achieve a good impedance matching between the SQUID array and the input and output lines. Second, the dispersion relation is engineered with a low plasma frequency of $30 \mathrm{GHz}$. Then the parasitic parametric processes coming from higher Bloch bands are strongly suppressed since they are poorly phase matched. As a last check, we compare the experimental data to the theoretical backward amplification [see Fig. 4(a)]. Again, theory and experimental data are in very good agreement.

\section{NOISE MEASUREMENTS}

Noise performance of the STWPA is estimated by measuring the noise power spectral density (PSD) at the output of the whole measurement chain, when the pump is 

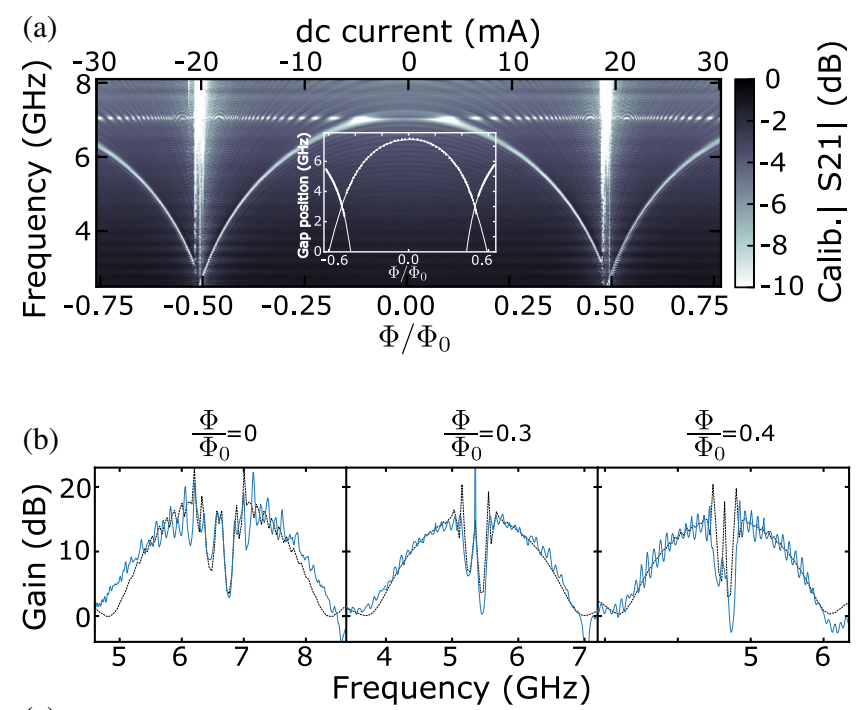

(c)

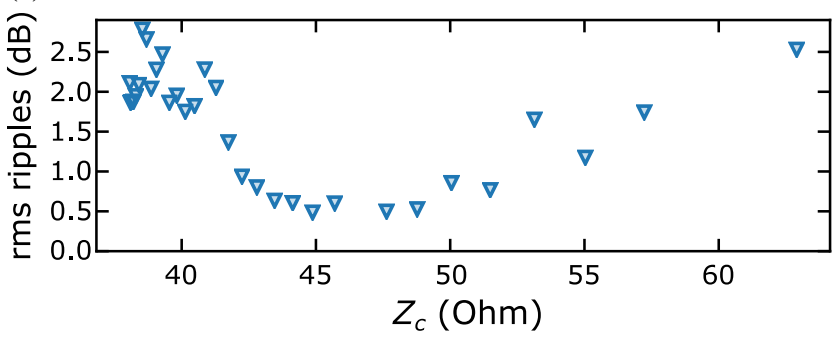

FIG. 5. (a) Calibrated transmission versus flux and probe frequency. Inset: interpolation of the gap position down to zero frequency. The distance between two intercrossing domains at zero frequency gives information about the SQUID stray inductance. (b) Three gain profiles of device $B$ for three different flux points. Gain ripples evolve with flux bias. (c) Root-mean-square values of gain ripples for 25 different flux biases. Each of this flux is converted to a characteristic impedance $\sqrt{\overline{(L)}(\Phi) / \bar{C}^{g}}$. A local minimum is found for a small-signal impedance equal to $48 \Omega$.

on and when it is off. We relate the measured noise PSD to the system noise $T_{\text {syst }}$ as

$$
\mathrm{PSD}=G_{\text {syst }} k_{B} T_{\text {syst }},
$$

where $G_{\text {syst }}$ is the system gain. We obtain $G_{\text {syst }}$ using an accurate calibration of the input line (see Sec. III). The system noise temperature is shown in Fig. 4(b). System noise has been measured during the same run as backward amplification with configuration (2). Unfortunately, the impedance matching was slightly worse than with configuration (1) and the gain flatness degraded. System noise has two main contributions, finite STWPA gain and intrinsic loss within the STWPA. The first one causes oscillations in $T_{\text {syst }}$. Indeed, when the STWPA gain decreases, the noise contribution of the following high electron mobility transistor (HEMT) amplifier increases. The second one directly affects the STWPA noise temperature since signal is lost while traveling inside the device.
STWPA intrinsic input noise, denoted $T_{\text {TWPA }}$, is plotted in Fig. 4(b) (brown dotted line, in kelvin). The system noise [black dotted line, Fig. 4(b)] is calculated as

$$
T_{\text {syst }}=T_{\mathrm{TWPA}}+T_{\text {syst,off }} / G_{\mathrm{TWPA}},
$$

where $T_{\text {syst,off }}$ is the measured system noise when the STWPA pump is off [green dotted line, Fig. 4(b)]. The system attenuation $A_{\text {system }}$ extracted previously from the theory leads to a system noise between 0.5 and $2 \mathrm{~K}$, depending on the STWPA gain. This system noise is explained by the STWPA intrinsic noise around $0.4 \mathrm{~K}$ (brown dashed line) just above the standard quantum limit (red dashed line) and the finite STWPA gain (see Sec. E).

We characterize the signal-to-noise ratio (SNR) improvement and gain compression using the line calibration described in Appendix D. SNR improvement measurement is carried out by sending a very low power signal into the system while probing the power spectral density with a spectrum analyzer with a bandwidth of $20 \mathrm{kHz}$ when the STWPA pump is on and off. Data are normalized with the total system gain. SNR improvement has been measured while the signal is sent on a gain peak and a gain dip. We observe an improvement between $15 \mathrm{~dB}$ (gain peak) and $10 \mathrm{~dB}$ (gain dip) in the SNR for an input signal $P_{\text {signal }}=-135 \mathrm{dBm}$ while we measure a $1-\mathrm{dB}$ compression point $P_{1 \mathrm{~dB}}=-100 \mathrm{dBm}$ for a $20 \mathrm{~dB}$ reference gain (see Appendix F).

\section{RESPONSE TO A FLUX OF THE SQUID TRANSMISSION LINE}

A useful and new feature of our STWPA is its flux tunability. It allows for the band gap to be tuned and hence the pump frequency. In situ modulation of the characteristic impedance $Z_{c}$ is also an interesting capability to mitigate the fabrication uncertainties, mainly regarding $\bar{L}$. We now report a detailed study of sample $B$ to illustrate such feature. A color map of its linear transmission as a function of frequency and $\Phi / \Phi_{0}$ is shown in Fig. 5(a). The photonic gap lies at $7 \mathrm{GHz}$ at zero flux and drops towards zero frequency for fluxes close to $\left|\Phi / \Phi_{0}\right|=0.5$. A jump in the gap position is visible when it goes below $4 \mathrm{GHz}$.

This jump is hysteretic: It occurs at a different flux whether the current is swept up or down [57]. This is a characteristic signature of a non-negligible ratio $\beta$ between $\bar{L}_{J J}$, the inductance of one junction of the SQUID and $L_{s}$, the stray inductance of the whole superconducting loop $\left(\beta=L_{s} / \bar{L}_{J J}\right)$. As shown in the inset of Fig. 5(a), by interpolating the gap position to low frequencies, we can infer the position of the crossing between the zero fluxoid domain and the \pm 1 fluxoid domain. Furthermore, there is a zone of coexistence between these domains. At zero frequency, the amplitude of this coexistence zone is noted $\Delta c=\Delta \Phi_{\mathrm{dc}} / \Phi_{0} . \quad \Delta c$ is directly proportional to the 
inductances ratio $\Delta c=\beta / 2$ [58]. From the interpolation we find $\Delta c=0.16$, and thus $\beta=0.32$. Taking into account the kinetic inductance of the wires connecting the two Josephson junctions forming the SQUID, we find a loop inductance $L_{s}=21 \mathrm{pH}$ [22]. This translates into a ratio $\beta=0.21$, which is on par with the measured one.

Our STWPA were designed to feature a characteristic impedance below $50 \Omega$ at zero flux. We then expect to observe a decrease of the gain ripples down to a local sweet spot, where the STWPA is perfectly matched to the external circuitry. Three amplification profiles taken at different flux are shown in Fig. 5(b). They all show 15-dB gain for a $2-\mathrm{GHz}$ bandwidth. It appears clearly that ripples are minimized for $\Phi / \Phi_{0}=0.3$. To quantitatively investigate impedance dependence of gain ripples, we measured gain profiles for more than 25 different magnetic fluxes from zero flux to $\Phi / \Phi_{0}=0.4$. Each profile was smoothed using Savitzky-Golay algorithm [59]. We then computed the averaged root-mean-square (rms) difference between the smoothed and experimental profile on a frequency band ranging between $11 \mathrm{~dB}$ and the maximum gain (the band corresponding to the gap is disregarded, see Appendix G for details). The rms ripples value is plotted versus linear impedance in Fig. 5(c). We observe the existence of a local minimum, in a interval between 45 and $48 \Omega$, clearly just below $50 \Omega$. This is expected as the Josephson inductance increases when pumped, and we use linear inductance to calculate flux dependent characteristic impedance. Ripples do not reach zero at the sweet spot because of remaining impedance mismatches between the setup and the STWPA.

\section{CONCLUSION}

In conclusion, we demonstrate a new design of SQUIDbased traveling-wave parametric amplifier. This amplifier is near quantum limited, compact, and directly compatible with superconducting qubits integration. It features high gain and large bandwidth despite its simple fabrication process. The absence of resonant phase matching is compensated by engineering the dispersion and opening a photonic band gap. A good understanding of the nonlinear behavior of this Josephson photonic crystal opens the door to further more fundamental experiments with such devices [60]. Finally, we demonstrate the possibility to implement TWPA made from SQUID, which could lead in the future to third-order Kerr nonlinearity TWPA, based on the SNAIL geometry [21,61-63] or to TWPA with vanishingly small fourth-order Kerr nonlinearity [64,65].

\section{ACKNOWLEDGMENTS}

The authors would like to thank L. Del Rey, D. Dufeu, E. Eyraud, and J. Jarreau for support with the experimental setup, M. Selvanayagam and D. Estève for critical reading of the manuscript, $\mathrm{P}$. David for help with the fabrication of the top ground, and V. Milchakov for the high quality sample pictures. The samples were fabricated in the Nanofab clean room located at Institut Néel. This research was supported by the ANR under contracts CLOUD (Project No. ANR-16-CE24-0005) and by the European Union's Horizon 2020 Research and Innovation Programme, under Grant agreement No. 824109, the European Microkelvin Platform (EMP). J. P. M. acknowledges support from the Laboratoire d'excellence LANEF in Grenoble (ANR-10-LABX-51-01). R. D. and S. L. acknowledge support from the CFM Foundation and the "Investisements d'avenir" (ANR-15-IDEX-02) programs of the French National Research Agency. A. R. acknowledges the European Union's Horizon 2020 research and innovation programme under the Marie Sklodowska-Curie Grant agreement No. 754303.

\section{APPENDIX A: FABRICATION PARAMETERS}

The STWPA is one long array of $N_{J}$ SQUID's. Each SQUID is made of two identical Josephson junctions, undergoing a sinusoidal modulation of their height $H$. Junctions are fabricated using a bridge-free technique [58]. This step is followed by the deposition of a 28 -nm-thick conformal layer of alumina via atomic layer deposition on top of the array. Finally, $1 \mu \mathrm{m}$ of copper is deposited on top of the alumina, working as a top metallic ground. These steps are shown schematically in Fig. 6(a). The inverted

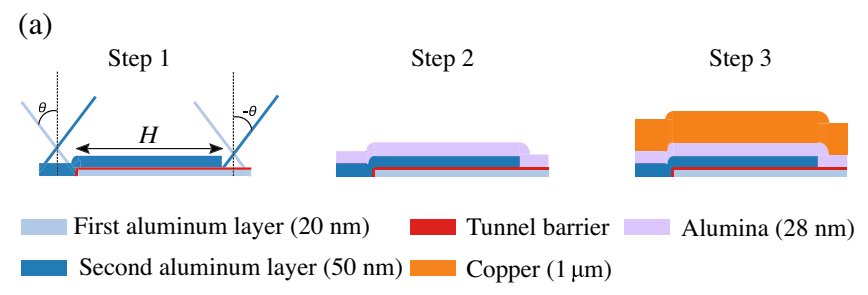

(b)

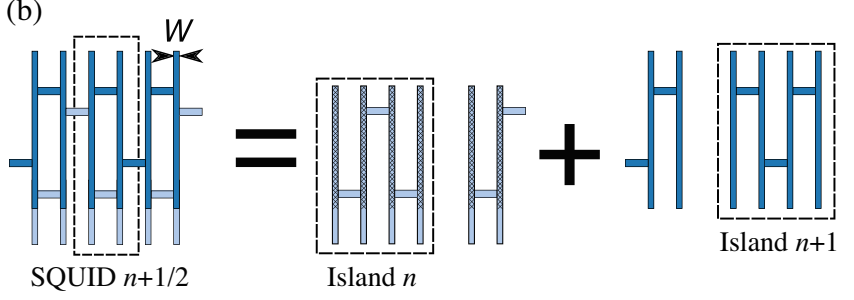

FIG. 6. Screening effect of the ground capacitance. (a) Longitudinal cut of a junction during the fabrication process. The first step is a double angle evaporation of aluminum via bridge-free technique with $\theta=35^{\circ}$. The overlap between the two layers define the height $H$ of a junction. Second step is a conformal deposition of alumina using atomic layer deposition. Third step is a sputtering of a thick copper layer. The bottom layer is mostly screened by the top layer. (b) Top view of the junctions. In this example, this short array is composed of three SQUID's labeled $n-1 / 2, n+1 / 2, n+3 / 2$, and two superconducting islands, $n$ and $n+1$. Regarding the superconducting island, the capacitance between the bottom layer and the top ground is much smaller than that between the top layer and the top ground as the hatched region of the bottom layer is totally screened by the top layer. 
stripline geometry with very close ground allows us to have a precise value of the ground capacitance $C^{g}$.

In our geometry, given the extreme closeness of the ground, the capacitance between the bottom layer and the ground is much smaller than that between the top layer and the ground. Thus, the two neighboring junctions do not have the same value of $C^{g}$. Nonetheless, the effect of this double periodicity can be discarded as it only affects the dispersion near the plasma frequency $(30 \mathrm{GHz})$ and we work at frequencies around $7 \mathrm{GHz}$. At such low frequencies, it is the ground capacitance per unit length that counts, so one can use the mean value of the two islands to determine the average $\bar{C}^{g}=42.6 \mathrm{fF}$. Furthermore, the region of the bottom layer which is not screened by the top layer, does not depend on the junction height $H$. Thus, it decreases the effective modulation amplitude of the ground capacitance $\zeta$.

The Josephson capacitance is taken as $45 \mathrm{fF}$ per square micrometer. The area of each junction is given by $H \times W$ (see Figs. 6(a) and 6(b)), and each SQUID consists of two junctions. As a result, the average SQUID has a Josephson capacitance $\bar{C}=485 \mathrm{fF}$.

Since Josephson inductance is exponentially sensitive to the tunnel junction thickness, the average inverse inductance $\bar{L}^{-1}$ is the most sensitive fabrication parameter whose value cannot be reliably determined from the geometry. Thus, it was determined by fitting the dispersion relation.

\section{APPENDIX B: THEORY OF THE JOSEPHSON PHOTONIC CRYSTAL TRAVELING-WAVE PARAMETRIC AMPLIFIER}

\section{Pump-wave propagation}

We assume the pump propagation to be unaffected by signal and idler, so it can be studied separately. The solution for the pump will then determine signal and idler propagation. We assume both the modulation amplitude and the nonlinearity strength to be weak; even in this case finding the pump wave represents a nontrivial problem when the pump frequency is close to the gap. The reason is that near the gap, the solution is given by a nonperturbative mixture of two plane waves, whose coefficients can be significantly affected even by a weak nonlinearity. As a result, the gap position in frequency depends on the amplitudes of both waves. These, in turn, depend on the pump power in a nontrivial way: for a fixed incident external power, some power is reflected back, while the power that enters the chain is sensitive to the dispersion inside the chain (e.g., the gap position), which, in turn, depends on the wave amplitudes inside the chain. Thus, the pump reflection at the chain end as well as its propagation inside the chain should be determined self-consistently for a given external incident pump power. This is quite different from the approach adopted in most studies on TWPA, where the spatial shape of the pump wave is assumed to be unaffected by the weak nonlinearity (in particular, see Ref. [36], where the Bloch functions of a spatially modulated amplifier were taken to be power independent).

We model the chain as a sequence of superconducting islands, labeled by an integer $n=0,1, \ldots, N$. The dynamical variables are the superconducting phases $\phi_{n}$ which satisfy the following equations of motion in the bulk of the chain:

$$
\begin{aligned}
& \frac{d^{2}}{d t^{2}}\left[C_{n}^{g} \phi_{n}+C_{n+1 / 2}\left(\phi_{n}-\phi_{n+1}\right)+C_{n-1 / 2}\left(\phi_{n}-\phi_{n-1}\right)\right] \\
& \quad+\frac{\sin \left(\phi_{n}-\phi_{n+1}\right)}{L_{n+1 / 2}}+\frac{\sin \left(\phi_{n}-\phi_{n-1}\right)}{L_{n-1 / 2}}=0 .
\end{aligned}
$$

Here, $C_{n}^{g}$ is the capacitance between the $n$th island and the ground, while $C_{n+1 / 2}$ and $L_{n+1 / 2}$ are the capacitance and the Josephson inductance of the junction between the islands $n$ and $n+1$ (hence the half-integer index). In the following, the Josephson nonlinearity will be expanded to the leading order, $\sin \theta \approx \theta-\theta^{3} / 6$.

The inductances and the capacitances are assumed to have a weak spatial modulation given by Eq. (1). It is convenient to introduce the plasma frequency $\omega_{\infty}=1 / \sqrt{\bar{L} \bar{C}}$ and the Coulomb screening length $\ell_{s}=\sqrt{\bar{C} / \bar{C}^{g}}$. Assuming the pump to be (i) monochromatic and (ii) a mixture of at most two resonant plane waves, we look for the solution of Eq. (B1) in the form

$$
\phi_{n}^{p}(t)=\left(A e^{i k n}+B e^{i k n-i G n}\right) e^{-i \omega_{p} t}+\text { c.c. },
$$

where $\omega_{p}$ is the pump frequency, determined by the external source, while the amplitudes $A, B$ and the wave vector $k$ should be determined from the equations; "c.c." stands for the complex conjugate. We assume $0<k<G$, substituting this expression in Eq. (B1), omitting high spatial and temporal harmonics, and approximating $\sin (k / 2) \approx k / 2$, $\sin (G / 2-k / 2) \approx G / 2-k / 2, \cos (k / 2) \approx \cos (G / 2-k / 2) \approx 1$, we obtain

$$
\begin{aligned}
& {\left[\frac{\omega_{p}^{2}}{\omega_{\infty}^{2} \ell_{s}^{2}}-k^{2}\left(1-\frac{\omega_{p}^{2}}{\omega_{\infty}^{2}}\right)+\frac{k^{4}}{2}|A|^{2}+k^{2}(G-k)^{2}|B|^{2}\right] A} \\
& \quad+\left[\frac{\zeta}{2} \frac{\omega_{p}^{2}}{\omega_{\infty}^{2} \ell_{s}^{2}}+\frac{\gamma}{2} k(G-k)\left(1-\frac{\omega_{p}^{2}}{\omega_{\infty}^{2}}\right)\right] B=0, \\
& {\left[\frac{\zeta}{2} \frac{\omega_{p}^{2}}{\omega_{\infty}^{2} \ell_{s}^{2}}+\frac{\gamma}{2} k(G-k)\left(1-\frac{\omega_{p}^{2}}{\omega_{\infty}^{2}}\right)\right] A} \\
& +\left[\frac{\omega_{p}^{2}}{\omega_{\infty}^{2} \ell_{s}^{2}}-(G-k)^{2}\left(1-\frac{\omega_{p}^{2}}{\omega_{\infty}^{2}}\right)+k^{2}(G-k)^{2}|A|^{2}\right. \\
& \left.+\frac{(G-k)^{4}}{2}|B|^{2}\right] B=0 .
\end{aligned}
$$

From these equations, one can obtain the linear dispersion relation by neglecting $|A|^{2}$ and $|B|^{2}$ inside the square 
brackets and looking for $k$ such that the obtained linear system for $A$ and $B$ admits a nonzero solution. In the nonlinear case, Eqs. (B3a) and (B3b) represent just two equations for three unknown quantities $k, A, B$. To close the equations, one should relate $A$ and $B$ to the incident power $P_{\text {in }}$, which represents another external control parameter, in addition to $\omega_{p}$.

To describe the incident microwave, we model the circuit to the left of the $n=0$ island as a linear $L C$ transmission line. Formally, this corresponds to taking the same equations (B1) and setting $C_{n}^{g} \rightarrow C_{\mathrm{TL}}, C_{n+1 / 2} \rightarrow 0$, $L_{n+1 / 2} \rightarrow L_{\mathrm{TL}}$, as well as linearizing $\sin \left(\phi_{n}-\phi_{n+1}\right) \rightarrow$ $\phi_{n}-\phi_{n+1}$ for all $n<0$. The transmission line impedance $Z_{\mathrm{TL}}$ is then given by $Z_{\mathrm{TL}}=\sqrt{L_{\mathrm{TL}} / C_{\mathrm{TL}}}$. The solution for $n \leq 0$ is the sum of the incident and reflected wave,

$$
\phi_{n<0}^{p}(t)=\left(A_{\mathrm{in}} e^{i k_{\mathrm{TL}} n}+A_{r} e^{-i k_{\mathrm{TL}} n}\right) e^{-i \omega_{p} t}+\text { c.c. },
$$

with $k_{\mathrm{TL}}=\omega_{p} \sqrt{L_{\mathrm{TL}} C_{\mathrm{TL}}}$. Solution (B2) being valid at $n \geq 0$, the continuity at $n=0$ requires

$$
A_{\text {in }}+A_{r}=A+B,
$$

while Eq. (B1) at $n=0$ gives

$$
\begin{aligned}
& \frac{i \omega_{p} L_{1 / 2}}{Z_{\mathrm{TL}}}\left(A_{\mathrm{in}}-A_{r}\right) \\
& =\left[\left(1-\frac{\omega_{p}^{2}}{\omega_{\infty}^{2}}\right)(1+\gamma)-\frac{|k A+(k-G) B|^{2}}{2}\right] \\
& \quad \times[i k A+i(k-G) B] .
\end{aligned}
$$

The pump reflection at $n=N$ is neglected.

Finally, using the relation between the voltage $V_{n}$ and the phase, $d \phi_{n} / d t=2 e V_{n} / \hbar$ (with $e>0$, the electron charge being $-e$ ), we express the incident power $P_{\text {in }}$ in terms of the amplitude $A_{\text {in }}$ :

$$
P_{\mathrm{in}}=\frac{2 \hbar^{2} \omega_{p}^{2}}{Z_{\mathrm{TL}}(2 e)^{2}}\left|A_{\mathrm{in}}\right|^{2}
$$

We can also determine the power carried by the pump wave inside the chain. Defining it as the rate of change of all energy to the right of the site $n=0$,

$$
\begin{aligned}
P= & \frac{d}{d t} \sum_{n=1}^{\infty} \frac{\hbar^{2}}{(2 e)^{2}} \frac{C_{n}^{g} \dot{\phi}_{n}^{2}+C_{n+1 / 2}\left(\dot{\phi}_{n}-\dot{\phi}_{n+1}\right)^{2}}{2} \\
& +\frac{d}{d t} \sum_{n=1}^{\infty} \frac{\hbar^{2}}{(2 e)^{2}} \frac{1-\cos \left(\phi_{n}-\phi_{n+1}\right)}{L_{n+1 / 2}}
\end{aligned}
$$

and using Eq. (B1), we obtain

$$
P=\frac{\hbar^{2}}{(2 e)^{2}} \dot{\phi}_{1}\left[C_{1 / 2}\left(\ddot{\phi}_{0}-\ddot{\phi}_{1}\right)+\frac{\sin \left(\phi_{0}-\phi_{1}\right)}{L_{1 / 2}}\right]
$$

Inserting solution (B2), we obtain

$$
\begin{aligned}
P= & 2 \omega_{p} E_{J}\left[(k-G / 2)|A+B|^{2}+(G / 2)\left(|A|^{2}-|B|^{2}\right)\right] \\
& \times\left[\left(1-\frac{\omega_{p}^{2}}{\omega_{\infty}^{2}}\right)(1+\gamma)-\frac{|k A+(k-G) B|^{2}}{2}\right] .
\end{aligned}
$$

Equations (B3a)-(B3e) form a closed system for five unknown quantities $A, B, A_{\text {in }}, A_{r}, k$. While $A_{\text {in }}$ and $A_{r}$ are straightforwardly eliminated using Eqs. (B3c) and (B3e), solution of the remaining three equations represents a nontrivial task. Depending on the parameters, these equations may have no solutions (corresponding to the nonlinear gap for the pump), or may have several solutions. Moreover, not all these solutions necessarily propagate in the right direction. Generally, it is not obvious a priori, in which direction the wave (B2) is propagating, since it contains both positive and negative wave vectors. In this situation, one should look where the energy is flowing. Namely, for each solution, Eq. (B6) can be evaluated, and solutions corresponding to $P<0$ must be discarded. Indeed, such solutions correspond to the energy propagating in the opposite direction (we inject the pump on the left end of the chain, and want the energy to propagate from left to right). We note that the nonlinear dispersion shown in Fig. 7 shows two physical solutions for a given frequency, in a certain frequency range. This is a manifestation of the nonperturbative nature of the self-consistent nonlinear problem: The situation with multiple solutions cannot be

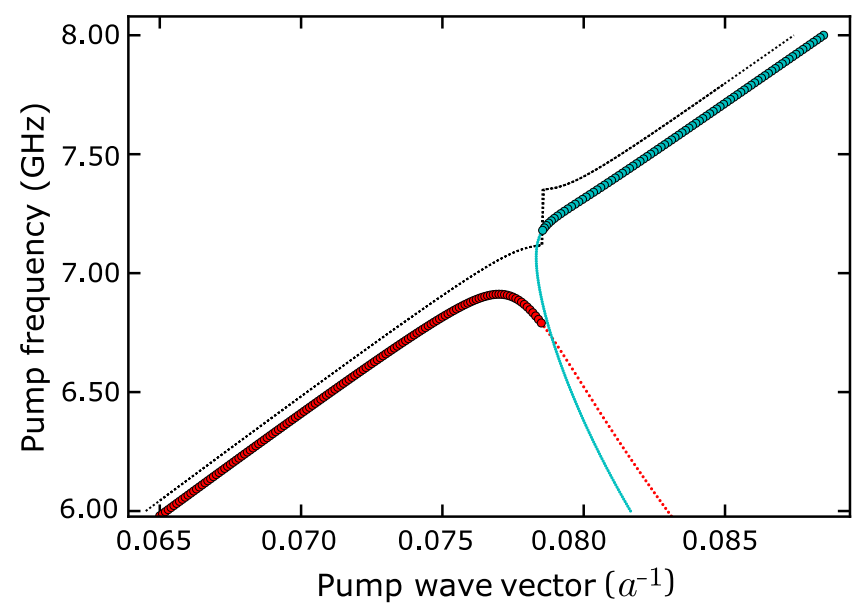

FIG. 7. Pump dispersion $\omega_{p}$ vs $k$ in the linear case (dashed line) and at $-71-\mathrm{dBm}$ incident power (symbols) determined by looking for solutions of Eqs. (A3a)-(A3e) for each pump frequency from 6 to $8 \mathrm{GHz}$. Thick symbols indicate the physical branches with $P>0$, thin symbols-unphysical branches with $P<0$. Note the existence of two physical solutions in a narrow interval of $\omega_{p}$ around $6.8 \mathrm{GHz}$. Investigation of their stability is beyond the scope of this paper. 
obtained by a smooth deformation of the single linear solution. A detailed study of possible solutions for the propagating pump and their stability represents a separate and nontrivial theoretical problem and is beyond the scope of the present paper. An even more complex problem would be to include the pump reflection at $n=N$. Indeed, the reflected pump wave would propagate at a (generally speaking) different wave vector, and it would give rise to the doubly reflected wave (from the $n=0$ ) as well as various nonlinear combinations. The consistent description of the system would then require the solution of $N$ nonlinear equations for all $\phi_{n}^{p}$ in the chain. Here, we solve the equations iteratively by varying $\omega_{p}$ and approaching the gap region either from higher or lower frequencies, thereby reconstructing the two branches of the power-dependent pump dispersion, as illustrated in Fig. 7.

\section{Signal and idler waves}

Having found the solution of Eq. (B1) for the pump in the form (B2), we add a weak perturbation oscillating at two other frequencies, $\omega_{s}$ (signal) and $\omega_{i}=2 \omega_{p}-\omega_{s}$ (idler):

$\phi_{n}(t)=\phi_{n}^{p}(t)+\left(\phi_{n}^{s} e^{-i \omega_{s} t}+\right.$ c.c. $)+\left(\phi_{n}^{i} e^{-i \omega_{i} t}+\right.$ c.c. $)$.

Substituting this expression into Eq. (B1), linearizing with respect to $\phi_{n}^{s}$ and $\phi_{n}^{i}$, and omitting high harmonics, we obtain a closed set of linear equations for $\phi_{n}^{s}$ and $\bar{\phi}_{n}^{i} \equiv\left(\phi_{n}^{i}\right)^{*}$ :

$$
\begin{aligned}
& \frac{\omega_{s}^{2}}{\omega_{\infty}^{2} \ell_{s}^{2}}(1+\zeta \cos G n) \phi_{n}^{s}+J_{n+1 / 2}^{s}-J_{n-1 / 2}^{s}=0, \\
& \frac{\omega_{i}^{2}}{\omega_{\infty}^{2} \ell_{s}^{2}}(1+\zeta \cos G n) \bar{\phi}_{n}^{i}+\bar{J}_{n+1 / 2}^{i}-\bar{J}_{n-1 / 2}^{i}=0
\end{aligned}
$$

where we introduced compact notations

$$
\begin{aligned}
J_{\nu}^{s} \equiv & (1+\gamma \cos G \nu) \\
& \times\left[\left(1-\frac{\omega_{s}^{2}}{\omega_{\infty}^{2}}-\left|\partial \phi_{\nu}^{p}\right|^{2}\right) \partial \phi_{\nu}^{s}-\frac{1}{2}\left(\partial \phi_{\nu}^{p}\right)^{2} \partial \bar{\phi}_{\nu}^{i}\right], \\
\bar{J}_{\nu}^{i} \equiv & (1+\gamma \cos G \nu) \\
& \times\left[\left(1-\frac{\omega_{i}^{2}}{\omega_{\infty}^{2}}-\left|\partial \phi_{\nu}^{p}\right|^{2}\right) \partial \bar{\phi}_{\nu}^{i}-\frac{1}{2}\left[\left(\partial \phi_{\nu}^{p}\right)^{*}\right]^{2} \partial \phi_{\nu}^{s}\right],
\end{aligned}
$$

and denoted $\partial \phi_{\nu} \equiv \phi_{\nu+1 / 2}-\phi_{\nu-1 / 2}$. These expressions with $\nu=1 / 2,3 / 2, \ldots, N-1 / 2$ define equations (B8a), (B8b) for $n=1,2, \ldots, N-1$. To close the system, we need two equations for $n=0$ and two more for $n=N$. Note that in the description of the pump wave no boundary condition at $n=N$ was invoked; this was because the pump reflection was neglected, so the propagation was the same as in a semi-infinite chain. For the signal and idler waves we want to include the reflection; even though weak due to impedance matching, the reflected waves might be amplified, and we want to account for this process.

As before, we model the circuit to the left of $n=0$ and to the right of $n=N$ as a linear $L C$ transmission line with the same parameters $L_{\mathrm{TL}}, C_{\mathrm{TL}}$. We assume that on the left we have a given incoming signal with amplitude $A_{\mathrm{in}}^{s}$, an unknown outgoing signal with amplitude $A_{\text {in }}^{s}-\phi_{0}^{s}$ (as follows from the continuity at $n=0$ ), zero incoming idler, and unknown outgoing idler with amplitude $\phi_{0}^{i}$, while on the right we have only outgoing signal and idler with unknown amplitudes $\phi_{N}^{s, i}$. This amounts to defining $J_{-1 / 2}^{s}$ and $J_{N+1 / 2}^{s, i}$ in Eqs. (B8a) and (B8b) for $n=0, N$ as

$$
\begin{gathered}
J_{-1 / 2}^{s} \rightarrow \frac{\partial \phi_{-1 / 2}^{s}}{L_{\mathrm{TL}} \omega_{\infty}^{2} \ell_{s}^{2} C_{g}}=\frac{i \omega_{s}}{\omega_{\infty} \ell_{s}} \frac{Z_{g}}{Z_{\mathrm{TL}}}\left[2 A_{\mathrm{in}}^{s}-\phi_{0}^{s}\right], \\
J_{-1 / 2}^{i} \rightarrow \frac{\partial \bar{\phi}_{-1 / 2}^{i}}{L_{\mathrm{TL}} \omega_{\infty}^{2} \ell_{s}^{2} C_{g}}=\frac{i \omega_{i}}{\omega_{\infty} \ell_{s}} \frac{Z_{g}}{Z_{\mathrm{TL}}} \bar{\phi}_{0}^{i}, \\
J_{N+1 / 2}^{s} \rightarrow \frac{\partial \phi_{N+1 / 2}^{s}}{L_{\mathrm{TL}} \omega_{\infty}^{2} \ell_{s}^{2} C_{g}}=\frac{i \omega_{s}}{\omega_{\infty} \ell_{s}} \frac{Z_{g}}{Z_{\mathrm{TL}}} \phi_{N}^{s}, \\
J_{N+1 / 2}^{i} \rightarrow \frac{\partial \bar{\phi}_{N+1 / 2}^{i}}{L_{\mathrm{TL}} \omega_{\infty}^{2} \ell_{s}^{2} C_{g}}=-\frac{i \omega_{i}}{\omega_{\infty} \ell_{s}} \frac{Z_{g}}{Z_{\mathrm{TL}}} \bar{\phi}_{N}^{i},
\end{gathered}
$$

where we define the impedances $Z_{\mathrm{TL}} \equiv \sqrt{L_{\mathrm{TL}} / C_{\mathrm{TL}}}, Z_{g} \equiv$ $1 /\left(\omega_{\infty} \ell_{s} C_{g}\right)$ of the transmission line and of the chain, respectively. Now the linear system (B8a), (B8b) is closed, so all $\phi_{n}^{s, i}$ can be found by the brute force numerical solution of this system. The resulting amplitudes are all proportional to $A_{\text {in }}^{s}$, so the ratios $\phi_{N}^{s} / A_{\text {in }}^{s}$ and $\left(A_{\text {in }}^{s}-\phi_{0}^{s}\right) / A_{\text {in }}^{s}$ give the signal amplification in transmission and reflection, respectively.

The described procedure is very general and does not make any a priori assumptions on which waves are amplified; waves propagating forward, or reflected back, or even evanescent (if the signal or idler frequency happens to be in the gap) are all treated on equal footing. We can afford this because the solution of a linear system, even a relatively large one $(N \sim 2000)$, can be obtained numerically in an efficient and reliable way. A general description of the pump wave including all reflections and combinations would require a solution of $N$ nonlinear equations, a much more complex numerical task. Thus, for the pump wave we neglect the reflection at $n=N$ and restrict ourselves to the simple form Eq. (B2).

To gain some qualitative insight into the amplification process, one should analyze the structure of the solution for $\phi_{n}^{s}$. In Fig. 8(a) we plot $\left|\phi_{n}^{s}\right|^{2}$, which shows the exponential growth in the forward direction, corresponding to the amplification. The wave content of the obtained solution can be better inferred via its spatial Fourier transform, 
(a)
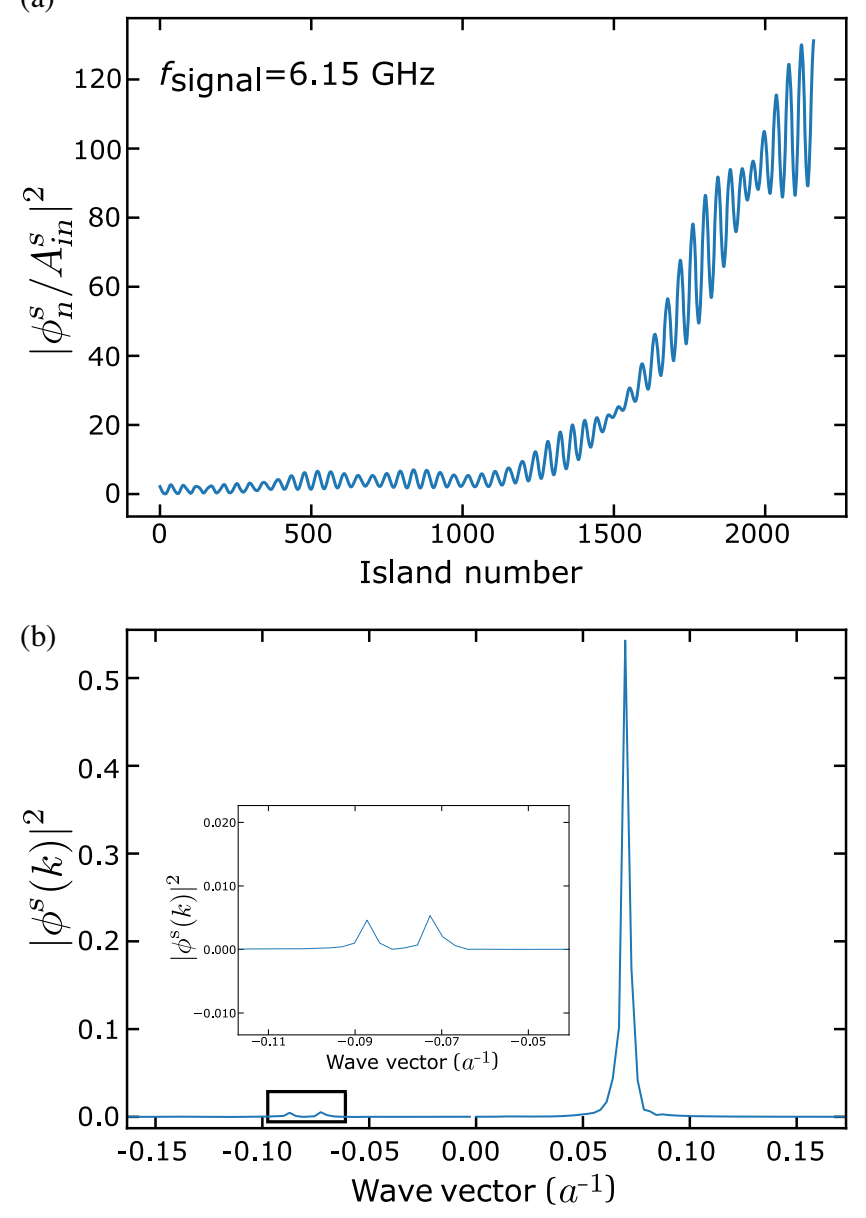

FIG. 8. (a) $\left|\phi_{n}^{\mathrm{s}}\right|^{2}$ for pump frequency equals to $6.901 \mathrm{GHz}$ and $-70 \mathrm{dBm}$ incident power. Signal frequency is $6.15 \mathrm{GHz}$. (b) Fourier transform $\left|\phi^{\mathrm{s}}(k)\right|^{2}$ of the signal shown in (a).

$$
\phi^{s}(k)=\sum_{n=0}^{N} \phi_{n}^{s} e^{-i k n} .
$$

We plot $\left|\phi^{s}(k)\right|^{2}$ Fig. 8(b). It shows three peaks, corresponding to the three components of the signal wave at $k_{s}>0,-k_{s}<0$, and $k_{s}-G<0$. The $-k_{s}$ component corresponds to the reflected wave.

It has been pointed out [45] that a TWPA with periodic spatial modulation is potentially subject to the parasitic effect of backward parametric amplification. Namely, if the pump wave has the profile $\phi_{n}^{p}=A_{p} e^{i k_{p} n}+B_{p} e^{i\left(k_{p}-G\right) n}$, and the amplification of the signal and idler waves with $k_{s}$, $k_{i}>0$ is efficient due to phase matching with the $k_{p} \approx G / 2$ component of the pump, $2 k_{p} \approx k_{s}+k_{i}$, this may automatically yield phase matching of $-k_{s},-k_{i}$ with the $k_{p}-G$ component of the same pump, $-k_{s}-k_{i} \approx 2\left(k_{p}-G\right)$, as shown schematically in Fig. 9.

To study possible phase-matched combinations, let us assume $\omega_{s}, \omega_{i}$ to be sufficiently far from the gap, so that one can straightforwardly define the signal and idler dispersion, corrected by the pump:

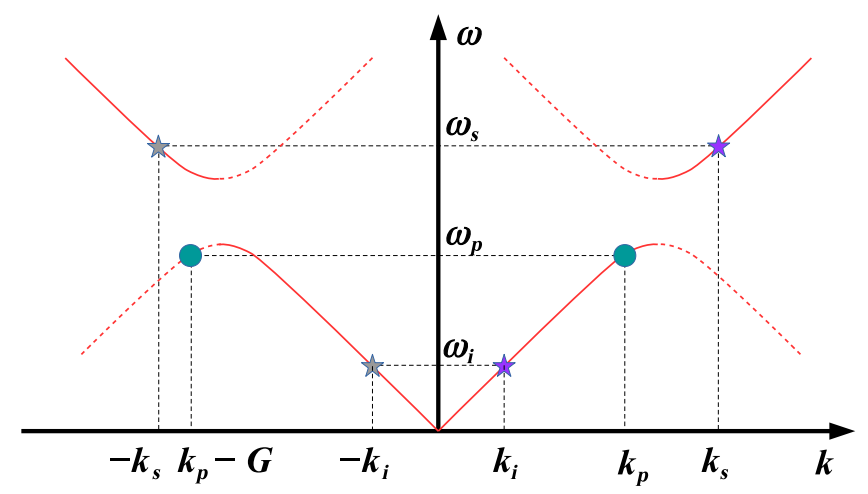

FIG. 9. A schematic view of the gapped dispersion (red curves, the dashed ones corresponding to folded dispersion) in the extended Brillouin zone picture. The green circles correspond to the two components of the pump wave with wave vectors $k_{p}>0$ and $k_{p}-G<0$, corresponding to the frequency $\omega_{p}$ near the gap edge. The purple stars represent signal and idler wave vectors $k_{s}, k_{i}>0$, corresponding to the frequencies $\omega_{s}, \omega_{i}$, while the gray stars represent $-k_{s},-k_{i}$, corresponding to the same frequencies. For $k_{p} \approx G / 2$, phase matching of $k_{s}, k_{i}$ with $2 k_{p}$ may automatically yield phase matching of $-k_{s},-k_{i}$ with $2\left(k_{p}-G\right)$ leading to potentially dangerous backward amplification.

$k_{s, i}^{2}=\frac{\omega_{s, i}^{2} /\left(\omega_{\infty} \ell_{s}\right)^{2}}{1-\omega_{s, i}^{2} / \omega_{\infty}^{2}-k_{p}^{2}\left|A_{p}\right|^{2}-\left(k_{p}-G\right)^{2}\left|B_{p}\right|^{2}}$.

This expression follows from Eqs. (B8) when terms proportional to $e^{i\left(k_{s}-G\right) n}, e^{i\left(k_{i}-G\right) n}$ are neglected. Wave vector mismatch for several combinations is plotted in Fig. 10. (When all frequencies are near the gap, it is not possible to define a simple expression for wave vectors and a single

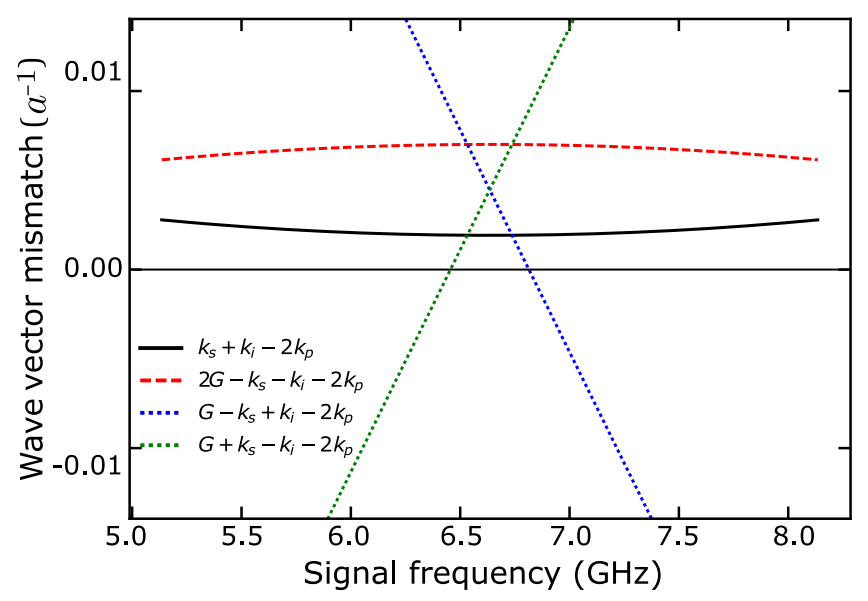

FIG. 10. Wave vector mismatch for four wave combinationscandidates for amplification. The solid line corresponds to the desired amplifier operation. The dashed line corresponds to the potential parasitic amplification of backward propagating signal and idler. The two dotted lines correspond to signal and idler propagating in opposite directions. Pump frequency and power are $6.901 \mathrm{GHz}$ and $-70 \mathrm{dBm}$, respectively. 
combination characterizing the phase mismatch, so the simple picture of Fig. 10 is not valid; still, the bandwidth of our TWPA well exceeds the gap width, so the assumption of $\omega_{s}, \omega_{i}$ being far from the gap is justified in most of the frequency interval.) From the figure it is seen that the standard forward amplification process has the smallest phase mismatch, while for the parasitic backward amplification it is significantly higher. This happens because the detuning of the pump-wave vector $k_{p}$ from $G / 2$ in our experiment, although small, $\left(\left|k_{p}-G / 2\right|=0.013\right)$, while $G / 2=0.0785$ is sufficient to suppress the phase matching for the backward amplification. This mismatch can also be understood graphically using Fig. 9. The forward process involves the green circle and the two purple stars. The three of them are well aligned. On the other hand, the backward amplification is described by the green circle and the gray stars. Their misalignment appears clearly.

\section{Gain ripples in a Fabry-Perot cavity with amplification}

The origin of the observed gain ripples and the difference in their amplitude between samples $A$ and $B$ can be understood from a very simple model without invoking the full theory of the previous subsection. Let us model the STWPA as a Fabry-Perot cavity formed by two identical mirrors whose reflection and transmission coefficients are real and given by $r$ and $t$, respectively, with $r^{2}+t^{2}=1$ [Fig. 11(a)]. The signal wave experiences multiple reflections inside the cavity, and we assume that upon each forward passage its amplitude is amplified by a (complex) factor $\Lambda$, while on the backward passage the amplification is neglected. In addition, each passage produces a phase factor $e^{i k_{s} N_{J}}$. The resulting total amplitude transmission of the whole structure is given by the sum over multiple reflections:

$$
\begin{aligned}
t_{\mathrm{tot}}= & t \Lambda e^{i k_{s} N_{J}} t+t \Lambda e^{i k_{s} N_{J}} r e^{i k_{s} N_{J}} r \Lambda e^{i k_{s} N_{J}} t \\
& +t \Lambda e^{i k_{s} N_{J}} r e^{i k_{s} N_{J}} r \Lambda e^{i k_{s} N_{J}} r e^{i k_{s} N_{J}} r \Lambda e^{i k_{s} N_{J}} t+\ldots \\
= & \frac{t^{2} \Lambda e^{i k_{s} N_{J}}}{1-r^{2} \Lambda e^{2 i k_{s} N_{J}}}
\end{aligned}
$$

The power gain is then given by

$\left|t_{\mathrm{tot}}\right|^{2}=\frac{t^{4}|\Lambda|^{2}}{1+r^{4}|\Lambda|^{2}-2 r^{2}|\Lambda| \cos \left(2 k_{s} N_{J}+\arg \Lambda\right)}$.

The ripples come from the cosine term in the denominator. It appears then clearly that their amplitude is higher for a larger gain.

To be quantitative, for $\Lambda$ we employ the standard expression [45]:
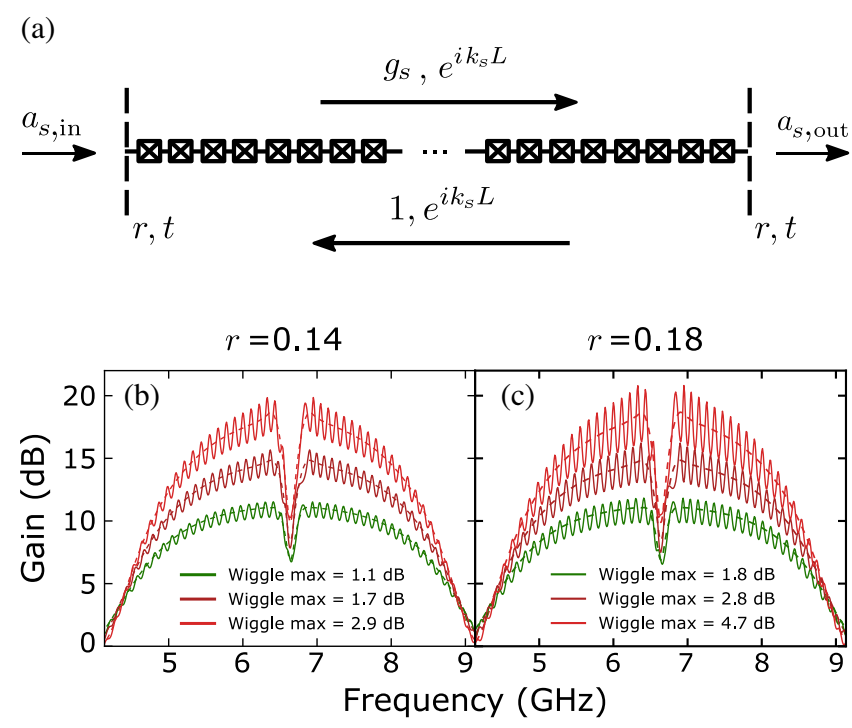

FIG. 11. (a) Modeling of the STWPA as a pumped Fabry-Perot cavity. Both input and output of the STWPA are modeled as semireflective mirrors with transmission coefficient $t$ and reflection coefficient $r\left(t^{2}+r^{2}=1\right)$. We consider that the counterpropagating signal is not amplified [see Fig. 4(a)]. (b) Signal power gain for different pump current $I_{p}$ (green: $I_{p}=0.4 I_{c}$, brown: $I_{p}=0.45 I_{c}$, and red: $\left.I_{p}=0.5 I_{c}\right)$. Larger pump current leads to higher maximum gain and larger ripples. The reflection coefficient is set to $r=0.14$ (c) Same parameters, but the reflection coefficient $r=0.18$. As expected, ripples have higher amplitude.

$$
\Lambda=\left(\cos g N_{J}-\frac{i \Delta k}{2 g} \sin g N_{J}\right) e^{i \Delta k N_{J} / 2}
$$

where $\Delta k=2 k_{p}-k_{s}-k_{i}$ is the mismatch of the wave vectors, determined by Eq. (B12). The gain coefficient $g=$ $\sqrt{\kappa_{s} \kappa_{i}^{*}-(\Delta k / 2)^{2}}$, where

$$
\kappa_{s}=\frac{\left(2 k_{p}-k_{i}\right) k_{s} k_{i}}{L C^{g} \omega_{s}^{2}} \frac{k_{p}^{2} Z_{\mathrm{TL}}^{2}}{16 L^{2} \omega_{p}^{2}} \frac{I_{p}^{2}}{I_{c}^{2}},
$$

$I_{p}$ is the peak current of the pump, $I_{c}$ is the SQUID critical current, and $\kappa_{i}$ is obtained by exchanging the subscripts $(s \leftrightarrow i)$. In Fig. 11(b), we set the reflection coefficient to $r=0.14$ and plot the signal power gain $\left|t_{\text {tot }}\right|^{2}$ for three different pump currents $I_{p}$. Naturally, higher pump currents produce larger maximum gain. We also observe an increase in the ripples amplitude. In Fig. 11(c), we set $r=0.18$ and plot the signal power gain for the same pump currents. The maximum gains are the same but ripples have a larger amplitude and we note the same dependence of their amplitude and the maximum gain on the pump current.

\section{APPENDIX C: EXPERIMENTAL SETUP}

The samples were measured with a vector network analyzer (model ZNB20 from Rohde \& Schwarz) in a 

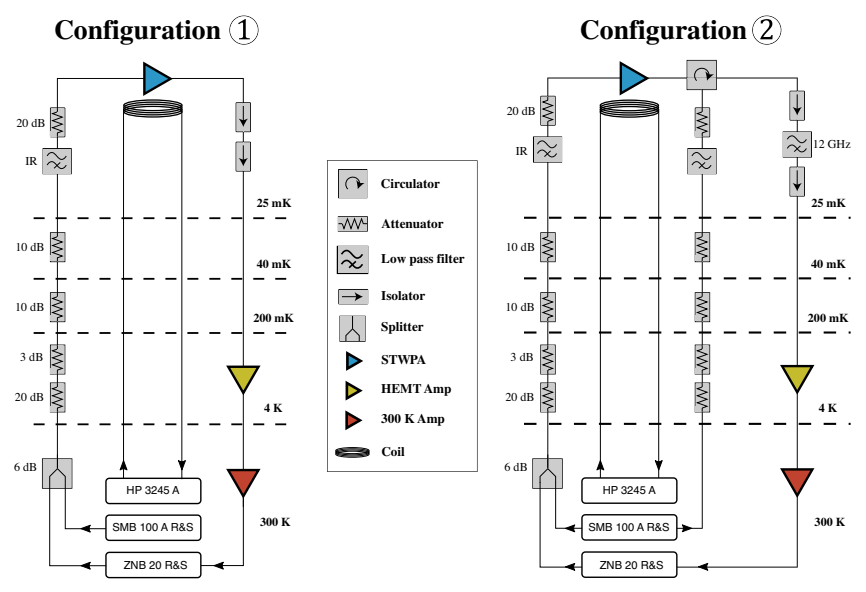

FIG. 12. Measurement setup. Configuration (1) is a standard microwave transmission measurement setup. Configuration (2) has one more input line. This configuration can be used for the same purpose as (1) (pump and signal propagate in the same direction), or to probe backward gain (pump and signal propagate in opposite directions). Attenuators and filter of the leftmost and middle rf lines are nominally identical.

dilution refrigerator with a base temperature of $25 \mathrm{mK}$. An additional microwave source (Rohde \& Schwarz SMB $100 \mathrm{~A}$ ) was used as a pump, while a global magnetic field was applied via an external superconducting coil powered by de current source (HP 3245 A). Both the coil and the sample were held inside a mu-metal magnetic shield. There are two measurement configurations: the first configuration, labeled (1), is a standard configuration where the STWPA is directly followed by two isolators and a commercial HEMT amplifier (model LNF-LNC1-12A from Low Noise Factory), whereas the second configuration, labeled (2), has a microwave circulator $(4 \mathrm{GHz}-8 \mathrm{GHz}$ ) between the output of the STWPA and the isolators to probe the backward gain, i.e., the amplification of any signal counterpropagating with respect to the pump direction (see Fig. 12). Configuration (2) can be used to measure both in a standard way (signal and pump propagating in the same direction) and backward gain (opposite directions). Experimental data shown on Figs. 2, 4(a), and 4(b) were taken with configuration (2). The rest was taken with configuration (1).

Attenuation and phase calibrations of the setup have been done by measuring a dummy $50-\Omega$ coplanar waveguide transmission line prior to the STWPA. This setup calibration protocol requires two cooldowns but is expected to be as precise as using an in situ microwave switch, since we have noticed that the attenuation in the lines differs by less than $0.5 \mathrm{~dB}$ from one cooldown to another.

\section{APPENDIX D: INPUT LINE CALIBRATION}

Calibration of the input line has been carried out by taking advantage of the power dependent cross-Kerr shift of the gap frequency position. We calibrated precisely the

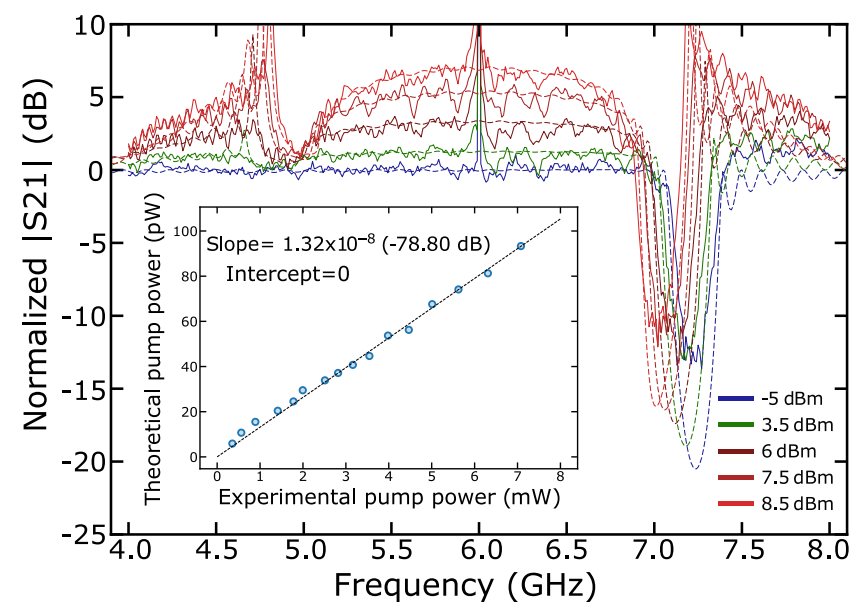

FIG. 13. Calibration via cross-Kerr shift. Comparison between experiment (full lines) and theory (dashed lines) for a pump tone sent at $6 \mathrm{GHz}$ at various power. This figure is similar to Fig. 2, but with more pump power, demonstrating the robustness of the model. Inset: experimental and theoretical pump power needed to get the same frequency shift of the gap. The linear fit gives a system attenuation $A_{\text {total }}=-78.8 \mathrm{~dB}$.

input line at $6 \mathrm{GHz}$, since we calibrated the added noise of the STWPA between 5.995 and $6.145 \mathrm{GHz}$. As shown in Sec. III, by sending a powerful pump tone at $6 \mathrm{GHz}$, we observe a red shift due to Kerr effect, as predicted by the theory. Since all the array parameters are set during the linear response study (fit of the linear dispersion relation), the only remaining free parameter to get an agreement between theory and experience is the pump power. Thus, by comparing the pump power needed to shift the gap by $\Delta f$ experimentally and theoretically, we can in principle infer the attenuation between the output of the pump source and the input of the STWPA. As shown in Fig. 13, by keeping the attenuation between the experimental and theoretical pump powers constant to $-78.8 \mathrm{~dB}$, we manage to reproduce very well the maximum gain, bandwidth, and frequency position of the gap. In the inset, we report the pump power needed to obtain the same gap frequency shift. We obtain a linear relation between the "hot" and "cold" pump power. The slope gives the total attenuation $A_{\text {total }}=-78.8 \mathrm{~dB}$.

As explained in Sec. III, this total attenuation takes into account the attenuation of the system $A_{\text {system }}$ and the attenuation due to the STWPA itself $A_{\text {TWPA }}$. In order to discriminate them, we have $A_{\mathrm{TWPA}}=\exp \left(k_{p}^{\prime \prime} N_{J} a\right)$, where $k_{p}^{\prime \prime}=\left(k_{p}^{\prime} \tan \delta\right) / 2$ is the imaginary part of the pump-wave vector calculated from our theory, $N_{J} a$ is the total length of the array and $\tan \delta$ is the high power loss tangent, inferred from experimental results. We find $A_{\mathrm{TWPA}}=-3.7 \mathrm{~dB}$.

\section{APPENDIX E: NOISE CANCELLATION}

In this section, we make explicit the methodology followed to get the input noise of the system composed 
(a)

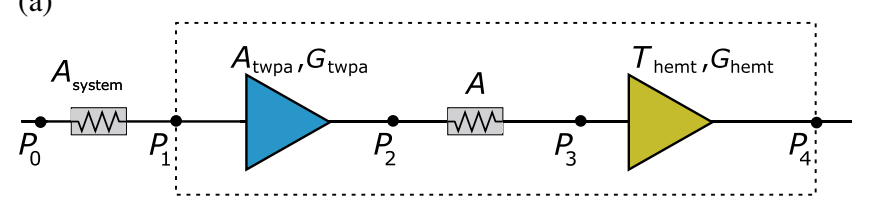

(b)

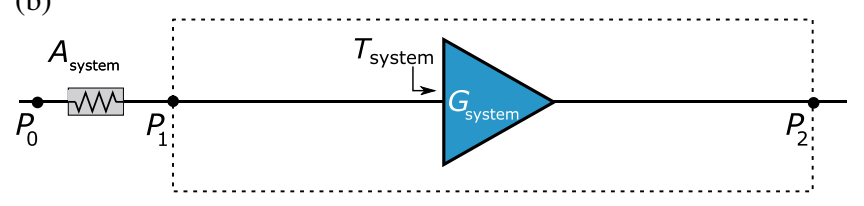

FIG. 14. Schematic representation of the system in terms of attenuators and amplifiers. (a) The system attenuation is $A_{\text {system }}$. Inside the dotted box, there is the STWPA, modeled as an attenuator combined with a lossless amplifier, followed by an unknown attenuation $A$ and the rest of the chain (HEMT and room-temperature amplifier), whose input noise is dominated by the HEMT noise. (b) Simpler modeling of the system as a single amplifier $G_{\text {system }}$, whose input noise $T_{\text {system }}$ depends on whether or not the STWPA pump is turned on.

of the STWPA, the HEMT amplifier, and the room-temperature amplifier. The reference plane for this system noise characterization is defined at the input of the STWPA, point $P_{1}$. We make the assumption that the noise at the system input $P_{1}$ [in Fig. 14(a)] is only made of quantum noise $N_{Q}$. We measure a PSD that equals the noise of the whole system by using a spectrum analyzer plugged at the system output $P_{4}$ [in Fig. 14(a)]. This system can be modeled as a single amplifier $G_{\text {system }}$ with an input noise $T_{\text {system }}$ [see Fig. 14(b)]. The total system gain $G_{\text {system }}$ is known by measuring the full transmission $\left|S_{21}\right|$ (at very low power), and subtracting the system attenuation $A_{\text {system }}$ from it (see Fig. 14). Whether the STWPA pump is on or off [blue and green lines, respectively, in Fig. 4(b)], we obtain a system noise close to the quantum limit or a system noise around $T_{\text {HEMT }}^{\prime}=15 \mathrm{~K}$. Two important points must be stressed. First, the system noise when the STWPA pump is off is higher than just the HEMT noise temperature $(3 \mathrm{~K})$ because of the attenuation between the HEMT and the system input, $A_{\text {TWPA }}$ and $A$. Second, the system noise does not reach the quantum limit when the STWPA is pumped. This is the consequence of losses within the STWPA and finite gain of the STWPA compared to the effective HEMT noise.

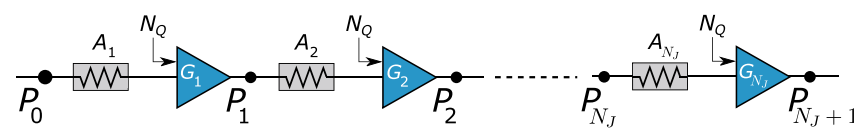

FIG. 15. Modeling of the STWPA as a cascade of attenuators and amplifiers. Every attenuator $A_{i}$ is equal to $A_{\mathrm{TWPA}, \mathrm{LP}} / N_{J}$ and every amplifier $G_{i}$ is equal to $G_{\mathrm{TWPA}} / N_{J}$. We make the assumption that every amplifier is quantum limited with an input noise $N_{Q}=1 / 2$.
We first calculate what would be the STWPA noise itself. We follow Macklin et al. [41] and model the STWPA as a cascade of $N_{J}$ cells made of an attenuator and an amplifier. Each attenuator is given by $A_{i}=A_{\mathrm{TWPA}, \mathrm{LP}} / N_{J}$ where $A_{\text {TWPA,LP }}=-5 \mathrm{~dB}$ is the attenuation within the STWPA for very low power, and each amplifier has a gain $G_{i}=$ $G_{\text {TWPA }} / N_{J}$ (see Fig. 15). In this model, every amplifier is quantum limited with an added noise of half a quanta $N_{Q}$ and we consider that the input noise of the first unit cell is quantum limited $\left(N_{0}=N_{Q}\right)$. At each point $P_{i}$, the noise in terms of added quanta is

$$
N_{i}=N_{i-1} A_{i} G_{i}+N_{Q}\left(1-A_{i}\right) G_{i}+N_{Q}\left(G_{i}-1\right) .
$$

Finally, the output STWPA noise at point $P_{N_{J}+1}$ can be normalized by the total transmission of the STWPA $G_{\text {TWPA }} \times A_{\text {TWPA }}$ and translated in a noise temperature denoted $T_{\text {TWPA }}$. We can therefore estimate the added noise by the STWPA alone [brown dotted line in Fig. 4(b)]. To take into account the second noise channel, namely the effective HEMT noise temperature, we simply consider the total system noise as being $T_{\text {system }}=T_{\text {TWPA }}+T_{\text {HEMT }}^{\prime} /$ $G_{\text {TWPA }}$, giving the black dotted line in Fig. 4(b). We identify why the system does not show strictly quantumlimited noise: internal dielectric loss and finite gain of the STWPA.

\section{APPENDIX F: SNR IMPROVEMENT AND GAIN COMPRESSION}

In this section, we show the SNR improvement of a signal sent at very low power $(-135 \mathrm{dBm})$ and frequency corresponding to a peak and a dip in the STWPA gain (6.1300 and $6.0700 \mathrm{GHz}$, respectively). We plot on the same figure (see Fig. 16) the PSD read with the spectral analyzer when the STWPA pump is on and off and normalize it by the total system gain (see Fig. 14). We observe a SNR improvement of $15 \mathrm{dBm}$ at a gain peak and $10 \mathrm{dBm}$ on a gain dip.

We also investigate the power saturation of the same STWPA, in the same configuration. Knowing the attenuation of the input line, we can infer the power required to compress the gain by $1 \mathrm{~dB}$. In Fig. 17, we plot the gain at a constant frequency $f_{\text {signal }}=6.130 \mathrm{GHz}$. We observe a gain increase at intermediate signal power and a drop for higher signal power. We believe this gain rise is caused by power dependent losses within the STWPA, which are decreasing when the signal power increases, as we also observed when the STWPA pump is off. An alternative explanation of this gain rise could be given. Indeed a similar effect was observed in resonant Josephson parametric amplifiers and explained by Kerr nonlinearity [20]. We do not have strong experimental proofs to discard either one of these explanations. The only clue is that we systematically observed a gain rise with increasing power and never a 

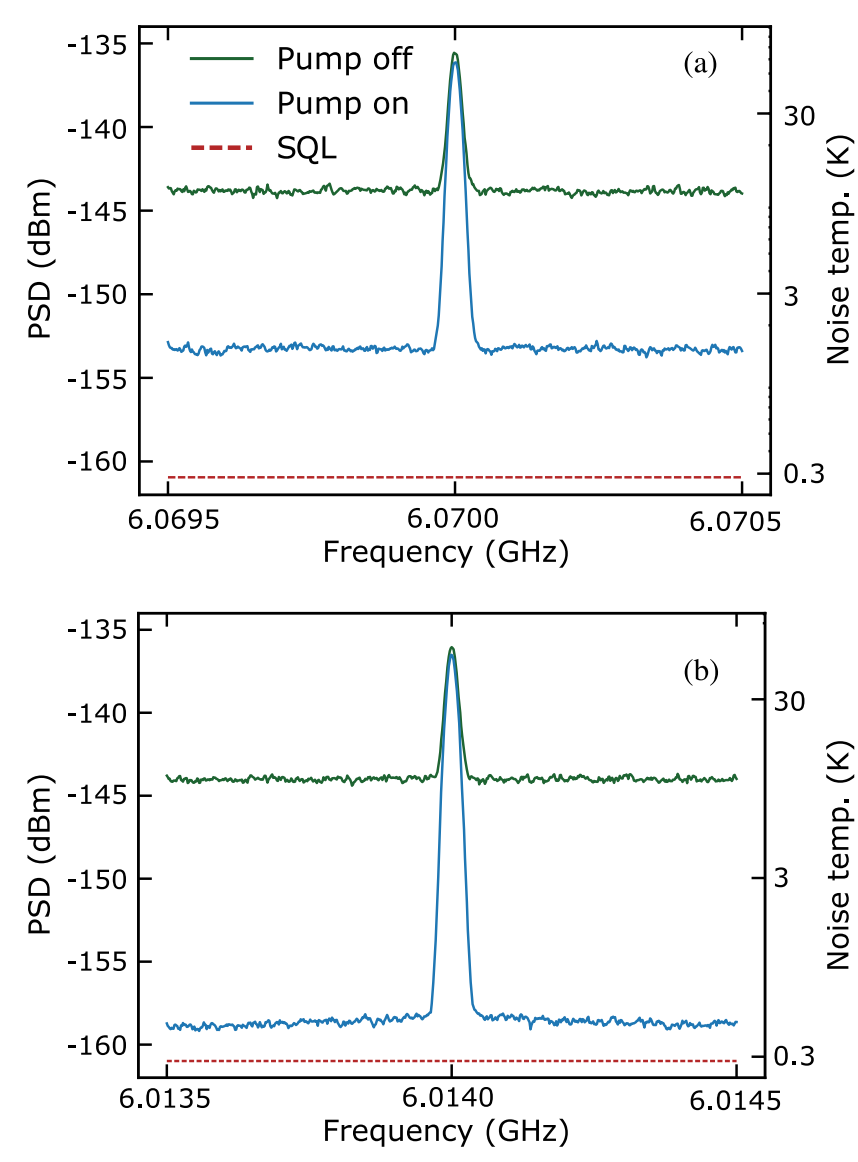

FIG. 16. SNR improvement. PSD normalized by the total system gain. An improvement of up to $15 \mathrm{dBm}$ is observed. Gain ripples lead to different SNR whether the signal frequency in on a gain peak or dip. (a) SNR improvement measured is on a gain dip. (b) SNR improvement measured on a gain peak.

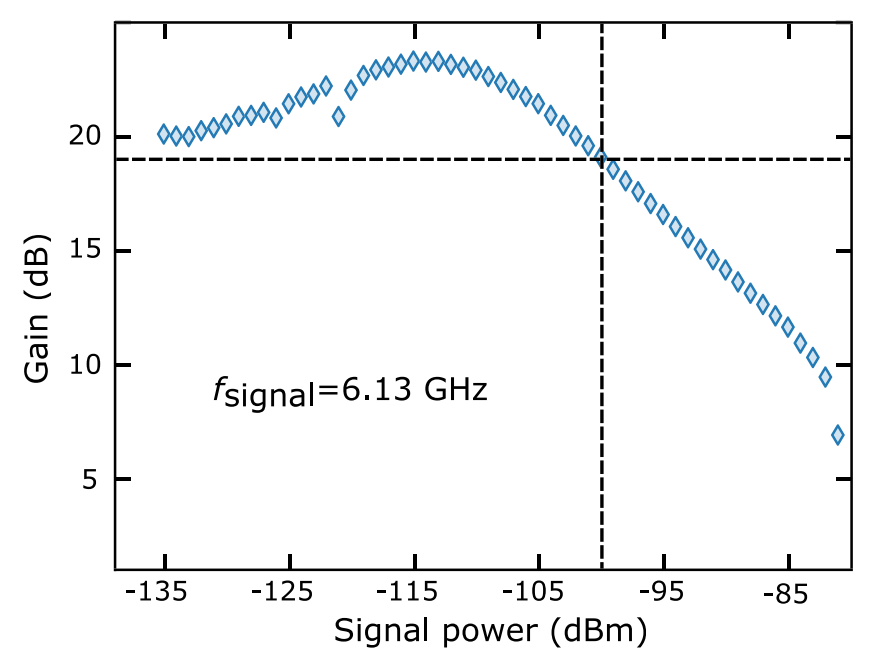

FIG. 17. Gain compression. Measured gain as a function of the signal power for $f_{\text {signal }}=6.13 \mathrm{GHz}$. The gain is compressed by $1 \mathrm{~dB}$ when the input signal power is equal to $-100 \mathrm{dBm}$.

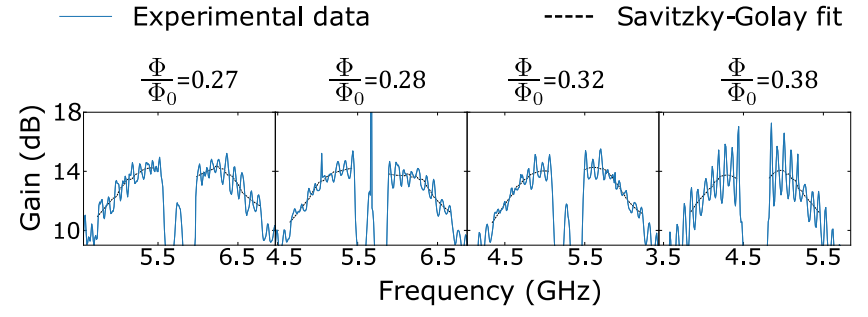

FIG. 18. Smoothing of several gain profiles using a SavitzkyGolay filter. As the maximum gain stays constant for all the fluxes, there is an improvement in the ripples as the characteristic impedance of the STWPA comes closer to $50 \Omega$. Note that the $y$-axis scale has a wider range for the upper row.

gain drop first, contrary to what is reported by Liu and coworkers [20]. For higher signal power, the gain starts to drop. We measure a $1-\mathrm{dB}$ compression point of $-100 \mathrm{dBm}$ for a $20-\mathrm{dB}$ reference gain (see Fig. 17).

\section{APPENDIX G: rms VALUE OF GAIN RIPPLES}

In this section, we explain how we extract the root-meansquare value of the gain ripples for sample $B$. As explained in Sec. VI, we fit the different gain profile with a SavitzkyGolay filter in order to smooth it, on a $-3-\mathrm{dB}$ band as shown in Fig. 18 for eight different magnetic fluxes. We then define the standard deviation as

$$
W=\sqrt{\frac{\sum_{f}\left(G_{\exp , f}-G_{\text {smooth }, f}\right)^{2}}{N} .}
$$

$W$ is plotted as the rms value of the gain ripples in the main text for more than 25 different gain profiles taken at different magnetic fluxes. The rms value is plotted as a function of the small-signal characteristic impedance $Z_{c}(\Phi)=\sqrt{\bar{L}(\Phi) / \bar{C}^{g}} \cdot \bar{C}^{g}$ is kept the same for all magnetic fluxes while $\bar{L}(\Phi)$ is inferred by fitting the dispersion relation for each corresponding flux.

[1] R. Vijay, D. H. Slichter, and I. Siddiqi, Observation of Quantum Jumps in a Superconducting Artificial Atom, Phys. Rev. Lett. 106, 110502 (2011).

[2] J. Stehlik, Y. Y. Liu, C. M. Quintana, C. Eichler, T. R. Hartke, and J. R. Petta, Fast Charge Sensing of a CavityCoupled Double Quantum Dot Using a Josephson Parametric Amplifier, Phys. Rev. Applied 4, 014018 (2015). 
[3] A. Bienfait, J. J. Pla, Y. Kubo, M. Stern, X. Zhou, C. C. Lo, C. D. Weis, T. Schenkel, M. L. W. Thewalt, D. Vion, D. Esteve, B. Julsgaard, K. Mølmer, J. J. L. Morton, and P. Bertet, Reaching the Quantum Limit of Sensitivity in Electron Spin Resonance, Nat. Nanotechnol. 11, 253 (2016).

[4] J. D. Teufel, T. Donner, D. Li, J. W. Harlow, M. S. Allman, K. Cicak, A. J. Sirois, J. D. Whittaker, K. W. Lehnert, and R. W. Simmonds, Sideband Cooling of Micromechanical Motion to the Quantum Ground State, Nature (London) 475, 359 (2011).

[5] P. K. Day, H. G. Leduc, B. A. Mazin, A. Vayonakis, and J. Zmuidzinas, A Broadband Superconducting Detector Suitable for Use in Large Arrays, Nature (London) 425, 817 (2003).

[6] N. Du et al., ADMX Collaboration, Search for Invisible Axion Dark Matter with the Axion Dark Matter Experiment, Phys. Rev. Lett. 120, 151301 (2018).

[7] H. Zimmer, Parametric Amplification of Microwaves in Superconducting Josephson Tunnel Junctions, Appl. Phys. Lett. 10, 193 (1967).

[8] B. Yurke, P. G. Kaminsky, R. E. Miller, and E. A. Whittaker, Observation of 4.2-K Equilibrium-Noise Squeezing via a Josephson-Parametric Amplifier, Phys. Rev. Lett. 60, 764 (1988).

[9] C. M. Caves, Quantum Limits on Noise in Linear Amplifiers, Phys. Rev. D 26, 1817 (1982).

[10] R. Movshovich, B. Yurke, P. G. Kaminsky, A. D. Smith, A. H. Silver, R. W. Simon, and M. V. Schneider, $O b-$ servation of Zero-Point Noise Squeezing via a JosephsonParametric Amplifier, Phys. Rev. Lett. 65, 1419 (1990).

[11] M. A. Castellanos-Beltran, K. D. Irwin, G. C. Hilton, L. R. Vale, and K. W. Lehnert, Amplification and Squeezing of Quantum Noise with a Tunable Josephson Metamaterial, Nat. Phys. 4, 929 (2008).

[12] N. Bergeal, F. Schackert, M. Metcalfe, R. Vijay, V. E. Manucharyan, L. Frunzio, D. E. Prober, R. J. Schoelkopf, S. M. Girvin, and M. H. Devoret, Phase-Preserving Amplification near the Quantum Limit with a Josephson Ring Modulator, Nature (London) 465, 64 (2010).

[13] J. Heinsoo, C. K. Andersen, A. Remm, S. Krinner, T. Walter, Y. Salathé, S. Gasparinetti, J.-C. Besse, A. Potočnik, A. Wallraff, and C. Eichler, Rapid High-Fidelity Multiplexed Readout of Superconducting Qubits, Phys. Rev. Applied 10, 034040 (2018).

[14] S. Kundu, N. Gheeraert, S. Hazra, T. Roy, K. V. Salunkhe, M. P. Patankar, and R. Vijay, Multiplexed Readout of Four Qubits in 3D Circuit QED Architecture Using a Broadband Josephson Parametric Amplifier, Appl. Phys. Lett. 114, 172601 (2019).

[15] A. Monfardini et al., NIKA: A Millimeter-Wave Kinetic Inductance Camera, Astron. Astrophys. 521, A29 (2010).

[16] J. Y. Mutus et al., Design and Characterization of a Lumped Element Single-Ended Superconducting Microwave Parametric Amplifier with On-Chip Flux Bias Line, Appl. Phys. Lett. 103, 122602 (2013).

[17] T. Roy, S. Kundu, M. Chand, A. M. Vadiraj, A. Ranadive, N. Nehra, M. P. Patankar, J. Aumentado, A. A. Clerk, and R. Vijay, Broadband Parametric Amplification with
Impedance Engineering: Beyond the Gain-Bandwidth Product, Appl. Phys. Lett. 107, 262601 (2015).

[18] O. Naaman, D. G. Ferguson, A. Marakov, M. Khalil, W. F. Koehl, and R. J. Epstein, High Saturation Power Josephson Parametric Amplifier with $\mathrm{GHz}$ Bandwidth, in Proceedings of the IEEE/MTT-S International Microwave SymposiumIMS 2019 (IEEE, Piscataway, NJ, 2019), pp. 259-262.

[19] C. Eichler and A. Wallraff, Controlling the Dynamic Range of a Josephson Parametric Amplifier, Eur. Phys. J. Quantum Technol. 1, 2 (2014).

[20] G. Liu, T. C. Chien, X. Cao, O. Lanes, E. Alpern, D. Pekker, and M. Hatridge, Josephson Parametric Converter Saturation and Higher Order Effects, Appl. Phys. Lett. 111, 202603 (2017).

[21] N. E. Frattini, V. V. Sivak, A. Lingenfelter, S. Shankar, and M. H. Devoret, Optimizing the Nonlinearity and Dissipation of a SNAIL Parametric Amplifier for Dynamic Range, Phys. Rev. Applied 10, 054020 (2018).

[22] L. Planat, R. Dassonneville, J. P. Martínez, F. Foroughi, O. Buisson, W. Hasch-Guichard, C. Naud, R. Vijay, K. Murch, and N. Roch, Understanding the Saturation Power of Josephson Parametric Amplifiers Made from SQUID Arrays, Phys. Rev. Applied 11, 034014 (2019).

[23] V. V. Sivak, N. E. Frattini, V. R. Joshi, A. Lingenfelter, S. Shankar, and M. H. Devoret, Kerr-Free Three-Wave Mixing in Superconducting Quantum Circuits, Phys. Rev. Applied 11, 054060 (2019).

[24] G. Agrawal, Nonlinear Fiber Optics, 5th ed., Optics and Photonics (Elsevier, Burlington, MA, 2012).

[25] A. S. Gilmour, Klystrons, Traveling Wave Tubes, Magnetrons, Crossed-Field Amplifiers, and Gyrotrons (Artech House, Boston, MA, 2011).

[26] A. L. Cullen, A Travelling-Wave Parametric Amplifier, Nature (London) 181, 332 (1958).

[27] V. V. Sivak, S. Shankar, G. Liu, J. Aumentado, and M. H. Devoret, Josephson Array-Mode Parametric Amplifier, Phys. Rev. Applied 13, 024014 (2020).

[28] P. Winkel, I. Takmakov, D. Rieger, L. Planat, W. HaschGuichard, L. Grünhaupt, N. Maleeva, F. Foroughi, F. Henriques, K. Borisov, J. Ferrero, A. V. Ustinov, W. Wernsdorfer, N. Roch, and I. M. Pop, Nondegenerate Parametric Amplifiers Based on Dispersion-Engineered Josephson-Junction Arrays, Phys. Rev. Applied 13, 024015 (2020).

[29] B. H. Eom, P. K. Day, H. G. Leduc, and J. Zmuidzinas, A. Wideband, Low-Noise Superconducting Amplifier with High Dynamic Range, Nat. Phys. 8, 623 (2012).

[30] C. Bockstiegel, J. Gao, M. R. Vissers, M. Sandberg, S. Chaudhuri, A. Sanders, L. R. Vale, K. D. Irwin, and D. P. Pappas, Development of a Broadband NbTiN Traveling Wave Parametric Amplifier for MKID Readout, J. Low Temp. Phys. 176, 476 (2014).

[31] M. R. Vissers, R. P. Erickson, H.-S. Ku, L. Vale, X. Wu, G. C. Hilton, and D. P. Pappas, Low-Noise Kinetic Inductance Traveling-Wave Amplifier Using Three-Wave Mixing, Appl. Phys. Lett. 108, 012601 (2016).

[32] A. A. Adamyan, S. E. de Graaf, S. E. Kubatkin, and A. V. Danilov, Superconducting Microwave Parametric Amplifier Based on a Quasi-Fractal Slow Propagation Line, J. Appl. Phys. 119, 083901 (2016). 
[33] S. Chaudhuri, D. Li, K. D. Irwin, C. Bockstiegel, J. Hubmayr, J. N. Ullom, M. R. Vissers, and J. Gao, Broadband Parametric Amplifiers Based on Nonlinear Kinetic Inductance Artificial Transmission Lines, Appl. Phys. Lett. 110, 152601 (2017).

[34] L. Ranzani, M. Bal, K. C. Fong, G. Ribeill, X. Wu, J. Long, H. S. Ku, R. P. Erickson, D. Pappas, and T. A. Ohki, Kinetic Inductance Traveling-Wave Amplifiers for Multiplexed Qubit Readout, Appl. Phys. Lett. 113, 242602 (2018).

[35] R. Landauer, Shock Waves in Nonlinear Transmission Lines and Their Effect on Parametric Amplification, IBM J. Res. Dev. 4, 391 (1960).

[36] R. P. Erickson and D. P. Pappas, Theory of Multiwave Mixing within the Superconducting Kinetic-Inductance TravelingWave Amplifier, Phys. Rev. B 95, 104506 (2017).

[37] E. A. Tholen, A. Ergul, E. M. Doherty, F. M. Weber, F. Gregis, and D. B. Haviland, Nonlinearities and Parametric Amplification in Superconducting Coplanar Waveguide Resonators, Appl. Phys. Lett. 90, 253509 (2007).

[38] A. J. Annunziata, D. F. Santavicca, L. Frunzio, G. Catelani, M. J. Rooks, A. Frydman, and D. E. Prober, Tunable Superconducting Nanoinductors, Nanotechnology 21, 445202 (2010).

[39] N. Maleeva, L. Grünhaupt, T. Klein, F. Levy-Bertrand, O. Dupre, M. Calvo, F. Valenti, P. Winkel, F. Friedrich, W. Wernsdorfer et al., Circuit Quantum Electrodynamics of Granular Aluminum Resonators, Nat. Commun. 9, 3889 (2018).

[40] N. Zobrist, B. H. Eom, P. Day, B. A. Mazin, S. R. Meeker, B. Bumble, H. G. LeDuc, G. Coiffard, P. Szypryt, N. Fruitwala, I. Lipartito, and C. Bockstiegel, Wide-Band Parametric Amplifier Readout and Resolution of Optical Microwave Kinetic Inductance Detectors, Appl. Phys. Lett. 115, 042601 (2019).

[41] C. Macklin, K. O’Brien, D. Hover, M. E. Schwartz, V. Bolkhovsky, X. Zhang, W. D. Oliver, and I. Siddiqi, A Near-Quantum-Limited Josephson Traveling-Wave Parametric Amplifier, Science 350, 307 (2015).

[42] M. Sweeny and R. Mahler, A Travelling-Wave Parametric Amplifier Utilizing Josephson Junctions, IEEE Trans. Magn. 21, 654 (1985).

[43] B. Yurke, M. L. Roukes, R. Movshovich, and A. N. Pargellis, A Low-Noise Series-Array Josephson Junction Parametric Amplifier, Appl. Phys. Lett. 69, 3078 (1996).

[44] O. Yaakobi, L. Friedland, C. Macklin, and I. Siddiqi, Parametric Amplification in Josephson Junction Embedded Transmission Lines, Phys. Rev. B 87, 144301 (2013).

[45] K. O'Brien, C. Macklin, I. Siddiqi, and X. Zhang, Resonant Phase Matching of Josephson Junction Traveling Wave Parametric Amplifiers, Phys. Rev. Lett. 113, 157001 (2014).

[46] T. C. White et al., Traveling Wave Parametric Amplifier with Josephson Junctions Using Minimal Resonator Phase Matching, Appl. Phys. Lett. 106, 242601 (2015).

[47] S. K. Tolpygo, V. Bolkhovsky, T. J. Weir, L. M. Johnson, M. A. Gouker, and W. D. Oliver, Fabrication Process and Properties of Fully-Planarized Deep-Submicron
$\mathrm{Nb} / \mathrm{Al}-\mathrm{AlO}_{x} / \mathrm{Nb}$ Josephson Junctions for VLSI Circuits, IEEE Trans. Appl. Supercond. 25, 1 (2015).

[48] L. Planat, E. Al-Tavil, J. P. Martínez, R. Dassonneville, F. Foroughi, S. Léger, K. Bharadwaj, J. Delaforce, V. Milchakov, C. Naud, O. Buisson, W. Hasch-Guichard, and N. Roch, Fabrication and Characterization of Aluminum Squid Transmission Lines, Phys. Rev. Applied 12, 064017 (2019).

[49] J. P. Martinez, S. Leger, N. Gheeraert, R. Dassonneville, L. Planat, F. Foroughi, Y. Krupko, O. Buisson, C. Naud, W. Hasch-Guichard, S. Florens, I. Snyman, and N. Roch, A Tunable Josephson Platform to Explore Many-Body Quantum Optics in Circuit-QED, npj Quantum Inf. 5, 19 (2019).

[50] C. Hutter, E. A. Tholén, K. Stannigel, J. Lidmar, and D. B. Haviland, Josephson Junction Transmission Lines as Tunable Artificial Crystals, Phys. Rev. B 83, 014511 (2011).

[51] M. Taguchi, D. M. Basko, and F. W. J. Hekking, Mode Engineering with a One-Dimensional Superconducting Metamaterial, Phys. Rev. B 92, 024507 (2015).

[52] A. Oliner and A. Hessel, Guided Waves on SinusoidallyModulated Reactance Surfaces, IRE Trans. Antennas Propag. 7, 201 (1959).

[53] N. W. Ashcroft and N. D. Mermin, Solid State Physics (Saunders College, Philadelphia, 1976).

[54] T. Weiß1, B. Küng, E. Dumur, A. K. Feofanov, I. Matei, C. Naud, O. Buisson, F. W. J. Hekking, and W. Guichard, Kerr Coefficients of Plasma Resonances in Josephson Junction Chains, Phys. Rev. B 92, 104508 (2015).

[55] Y. Krupko, V. D. Nguyen, T. Weissl, E. Dumur, J. Puertas, R. Dassonneville, C. Naud, F. W. J. Hekking, D. M. Basko, O. Buisson, N. Roch, and W. Hasch-Guichard, Kerr Nonlinearity in a Superconducting Josephson Metamaterial, Phys. Rev. B 98, 094516 (2018).

[56] R. Vijay, M. H. Devoret, and I. Siddiqi, Invited Review Article: The Josephson Bifurcation Amplifier, Rev. Sci. Instrum. 80, 111101 (2009).

[57] S. Pogorzalek, K. G. Fedorov, L. Zhong, J. Goetz, F. Wulschner, M. Fischer, P. Eder, E. Xie, K. Inomata, T. Yamamoto, Y. Nakamura, A. Marx, F. Deppe, and R. Gross, Hysteretic Flux Response and Nondegenerate Gain of FluxDriven Josephson Parametric Amplifiers, Phys. Rev. Applied 8, 024012 (2017).

[58] F. Lecocq, Quantum Dynamics in a dcSQUID: From the Phase Qubit to the 2D Quantum Oscillator, Ph.D. thesis, Université de Grenoble, 2011.

[59] A. Savitzky and M. J. E. Golay, Smoothing and Differentiation of Data by Simplified Least Squares Procedures, Anal. Chem. 36, 1627 (1964).

[60] A. L. Grimsmo and A. Blais, Squeezing and Quantum State Engineering with Josephson Travelling Wave Amplifiers, npj Quantum Inf. 3, 20 (2017).

[61] A. B. Zorin, Josephson Traveling-Wave Parametric Amplifier with Three-Wave Mixing, Phys. Rev. Applied 6, 034006 (2016).

[62] A. B. Zorin, M. Khabipov, J. Dietel, and R. Dolata, Traveling-Wave Parametric Amplifier Based on Three-Wave 
Mixing in a Josephson Metamaterial, in Proceedings of the 2017 16th International Superconductive Electronics Conference (ISEC) (IEEE, Piscataway, NJ, 2017), pp. 1-3.

[63] N. E. Frattini, U. Vool, S. Shankar, A. Narla, K. M. Sliwa, and M.H. Devoret, 3-Wave Mixing Josephson Dipole Element, Appl. Phys. Lett. 110, 222603 (2017).
[64] M. T. Bell and A. Samolov, Traveling-Wave Parametric Amplifier Based on a Chain of Coupled Asymmetric Squids, Phys. Rev. Applied 4, 024014 (2015).

[65] W. Zhang, W. Huang, M. E. Gershenson, and M. T. Bell, Josephson Metamaterial with a Widely Tunable Positive or Negative Kerr Constant, Phys. Rev. Applied 8, 051001 (2017). 